\title{
A 2'-O-Methyltransferase Responsible for Transfer RNA Anticodon Modification Is Pivotal for Resistance to Pseudomonas syringae DC3000 in Arabidopsis
}

\author{
Vicente Ramírez,, ${ }^{1}$ Beatriz González, ${ }^{1}$ Ana López, ${ }^{1,2}$ Maria Jose Castelló, ${ }^{1}$ Maria José Gil,, ${ }^{1}$ Bo Zheng, ${ }^{3}$ \\ Peng Chen, ${ }^{4}$ and Pablo Vera ${ }^{1,+}$ \\ ${ }^{1}$ Instituto de Biología Molecular y Celular de Plantas, Universidad Politécnica de Valencia-C.S.I.C, Ciudad Politécnica de la \\ Innovación, Edificio 8E, Valencia, Spain; ${ }^{2}$ Institute for Translational Plant and Soil Biology, Department of Animal and Plant \\ Sciences, The University of Sheffield, Sheffield, U.K.; ${ }^{3}$ College of Horticulture and Forestry Sciences, Huazhong Agricultural \\ University, Wuhan, China; and ${ }^{4}$ National Key Laboratory of Crop Genetic Improvement and National Centre of Plant Gene \\ Research, HuaZhong Agricultural University, Wuhan, China
}

Accepted 2 July 2018.

\begin{abstract}
Transfer RNA (tRNA) is the most highly modified class of RNA species in all living organisms. Recent discoveries have revealed unprecedented complexity in the tRNA chemical structures, modification patterns, regulation, and function, suggesting that each modified nucleoside in tRNA may have its own specific function. However, in plants, our knowledge of the role of individual tRNA modifications and how they are regulated is very limited. In a genetic screen designed to identify factors regulating disease resistance in Arabidopsis, we identified SUPPRESSOR OF CSB3 9 (SCS9). Our results reveal SCS9 encodes a tRNA methyltransferase that mediates the $2^{\prime}$ - $O$-ribose methylation of selected tRNA species in the anticodon loop. These SCS9-mediated tRNA modifications enhance susceptibility during infection with the virulent bacterial pathogen Pseudomonas syringae DC3000. Lack of such tRNA modification, as observed in $\operatorname{scs} 9$ mutants, specifically dampens plant resistance against DC3000 without compromising the activation of the salicylic acid signaling pathway or the resistance to other biotrophic pathogens. Our results support a model that gives importance to the control of certain tRNA modifications for mounting an effective disease resistance in Arabidopsis toward DC3000 and, therefore, expands the repertoire of molecular components essential for an efficient disease resistance response.
\end{abstract}

Transfer RNAs (tRNAs) are noncoding RNAs responsible for the addition of amino acids to the C-terminus of translating proteins. These molecules are 75 to 78 nucleotides long, with a characteristic three-dimensional L-shape, usually represented

Vicente Ramírez and Beatriz González contributed equally to this work.

${ }^{\dagger}$ Corresponding author: Pablo Vera; E-mail: vera@ibmcp.upv.es

Funding: Funding for this work were supported by Generalitat Valenciana, grant Prometeo2014/024 and MINECO, grant BFU2015-68199-R.

*The $\boldsymbol{e}$-Xtra logo stands for "electronic extra" and indicates that seven supplementary figures and three supplementary tables are published online.

This article is in the public domain and not copyrightable. It may be freely reprinted with customary crediting of the source. The American Phytopathological Society, 2018. in the form of a cloverleaf displaying the four distinctive stemloop arms, the acceptor arm, the variable D arm, the anticodon arm, and the variable/T $\Psi \mathrm{C}$ arm, which are generated by internal base pairing. A distinctive feature of tRNA is that, among the 107 posttranscriptional modifications found in RNA, 92 of these modified nucleosides are present on tRNA molecules that are involved in the mature tRNA processing required for its function (Machnicka et al. 2013). Interestingly, the biosynthetic pathway of the majority of tRNA modifications include at least a methylation step, and the number of modified bases varies among individual tRNA types and among different prokaryote and eukaryote species (Machnicka et al. 2014). Modifications vary from a single methylation on the ribose to more complicated modifications at different positions on the purine or pyrimidine ring (El Yacoubi et al. 2012; Hori 2014; Machnicka et al. 2013). Comprehensive information on structures and positions of these modifications in tRNA molecules, tRNA biosynthetic pathways, and responsible enzymes are deposited in different RNA modification databases (e.g., the MODOMICS database). Nucleoside tRNA modifications remain extensively studied in bacteria and yeast, and data has most recently been reported in humans. The fact that posttranscriptional modifications are highly conserved, based on the variety of organisms reported, underscores their importance. In bacteria and Saccharomyces cerevisiae, the relationship between the type of modification in tRNAs and metabolism has been suggested as a regulatory device acting as biological sensors, continually adjusting based on growth conditions (El Yacoubi et al. 2012; Novoa et al. 2012; Phizicky and Hopper 2010). However, little is documented on tRNAmodified nucleosides in plants, and research in genetic and biochemical regulation is still in its infancy. However, a recent study performed in Arabidopsis and hybrid aspen (Populus tremula $\times$ tremuloides) identified 21 modified nucleosides in tRNAs in both species (Chen et al. 2010). In addition, very few plant tRNA sequences are available to identify modified nucleosides on different positions of individual tRNA species, and information on plant tRNA modifying enzymes and the genetic characterization of mutant strains defective in specific tRNA modifications is still scant (Burgess et al. 2015; Chen et al. 2010; David et al. 2017; Kalhor and Clarke 2003; Miyawaki et al. 2006; Walden et al. 1982; Wang et al. 2017; Zhou et al. 2013).

From a compilation of tRNA sequences, Jühling et al. (2009) estimated that the cytoplasmic tRNAs of $S$. cerevisiae contain, on average, 12.6 modifications per tRNA molecule and, 
in particular, 2.6 of these modifications occur within the anticodon loop $\left(\mathrm{N}_{32}-\mathrm{N}_{38}\right)$. The rest of the modifications occur in the main body of the tRNA and appear crucial for folding and stability of the molecule, with the absence of certain body modifications eliciting a quality control pathway that results in the degradation of specific tRNAs at different stages of biosynthesis (Thompson and Parker 2009). Instead, many tRNA modifications that occur in the anticodon loop play several crucial roles in translation; deficiencies in modified nucleosides, like those influencing decoding of certain codons, lead to reduced translation efficiency and increased errors, ultimately affecting gene expression and cell metabolism (Agris et al. 2007; Björk 1995; Chan et al. 2012; Chen et al. 2010; Dedon and Begley 2014; Gu et al. 2014; Phizicky and Hopper 2010). As proposed by Chan et al. (2010, 2012), in response to external cues, nucleoside modification changes may be used as a "regulatory module" to timely adapt the cell to environmental changes in a fast, broad, and effective manner. In the majority of cases, however, it remains unclear exactly which tRNAs are specifically affected by a lack of specific modification and how the absence of specific tRNA modifications conferred specific phenotypes. One such crucial modification of a tRNA is the concurrent $2^{\prime}-O$-methylation of N32 and N34. The latter occupies the anticodon wobble nucleotide, which forms $\mathrm{Nm} 32$ and $\mathrm{Nm} 34$ in the anticodon loop. In yeast, $2^{\prime}-O$-methylation occurs on C32 and N34 of tRNA ${ }^{\text {Phe }}$, tRNA $^{\text {Leu }}$, and tRNA ${ }^{\text {Trp }}$, and such covalent modifications are mediated by the methyltransferase (MTase) Trm7p (Guy et al. 2012; Pintard et al. 2002); interestingly trm 7 mutant strains show growth reduction, presumably due to translation defects (Pintard et al. 2002). These 2'-O-methylations of the anticodon loop are highly conserved in eukaryotes and, in humans, defects in such modifications are associated with mental disorders (Kirchner and Ignatova 2015; Kuchino et al. 1982; Takano et al. 2008). Despite these observations in yeast and humans, no evidence exists, to date, supporting the importance of this tRNA modification type in plants and the consequences of a failure in its control by TRM7 homologous genes (Burgess et al. 2016).

The present study provides insights into the functional implications that some specific tRNA modifications at the anticodon loop had on the activation of an efficient diseaseresistance response in Arabidopsis toward the bacterial pathogen Pseudomonas syringae DC3000 (DC3000), the causal agent of bacterial speck disease. During a search for genetic suppressors in Arabidopsis for the enhanced disease-resistance csb3 mutant (Gil et al. 2005), we identified the SCS9 gene (standing for suppressor of $c s b 3$ ) encoding a functional homolog of the yeast MTase Trm7p. Our results provide evidence that SCS9 is crucial to maintain intact plant resistance toward DC3000. We employed genetic, molecular, and physiological analyses, and concluded it is vital to maintain an intact $2^{\prime}-O$ methylation of the tRNA anticodon loop molecules to ensure plant resistance against DC3000. Therefore, our results uncover a new critical component required for disease resistance in Arabidopsis.

\section{RESULTS}

\section{scs9 identification.}

The csb3 Arabidopsis-enhanced disease-resistant mutant was isolated during a search for negative disease-resistance regulators to biotrophic pathogens (Gil et al. 2005). Enhanced disease resistance to DC3000 occurred in $c s b 3$ plants, with increased accumulation of salicylic acid (SA) and constitutive expression of pathogenesis-related $(P R)$ genes. Moreover, epistasis analysis with different genes involved in the SA signal transduction pathway revealed $c s b 3$ plants required intact SA synthesis and its recognition through the NPR1 receptor (Gil et al. 2005).

We conducted a screen for $c s b 3$ suppressors in a second-site mutagenesis to further our understanding of plant immunity. Hence, homozygous $c s b 3$ seeds were mutagenized with ethyl methanesulfonate (EMS), and approximately 20,000 M2 seeds were grown and screened to identify individuals that no longer exhibited enhanced resistance to DC3000 attributable to the $c s b 3$ mutation. This screen rendered the isolation of the recessive $\operatorname{scs} 9$ (standing for suppressors of $c s b 3$ ) mutant (Fig. 1). $\operatorname{csb} 3 \operatorname{scs} 9$ plants lost the distinguishable morphological and growth-arrest phenotype of $c s b 3$ plants (Fig. 1A). The scs 9 mutant, when segregated from $c s b 3$, retained only a partial retardation in growth when compared with full-grown Col-0 plants (Fig. 1D). Such retardation in growth in the mutant could be observed from early stages of seedling development and accounted for a $20 \%$ reduction in fresh weight when compared with Col-0 (Supplementary Fig. S1). Also, the pathogen-responsive marker pP69C::GUS gene (Jordá and Vera 2000), constitutively expressed in the single csb3 mutant (Gil et al. 2005), was abrogated in the double $\operatorname{csb} 3 \operatorname{scs} 9$ mutant (Fig. 1B). Moreover, comparative observations of DC3000 growth rates in Col-0, csb3, and $\operatorname{csb3} \operatorname{scs} 9$ infected leaves showed the $\operatorname{scs} 9$ mutation blocks the characteristic enhanced resistance to DC3000 due to the csb3 mutation (Fig. 1C). Notably, $\operatorname{csb3} \operatorname{scs} 9$ plants were far more susceptible to DC3000 than Col-0 plants, and the observed enhanced susceptibility was of a similar magnitude, if not higher, to that observed in $n p r l$ plants (Fig. 1C). Furthermore, genetic analyses indicated the scs 9 mutation was extragenic to $c s b 3$ and, when segregated from the $c s b 3$ mutant background by backcrossing to Col0 plants, the compromised immune response conferred by the scs 9 mutation alone was maintained in the absence of the $\operatorname{csb} 3$ mutation (Fig. 1C). The enhanced bacterial growth in the single $\operatorname{scs} 9$ mutant was accompanied by distinguishable symptoms of induced chlorosis, presumably due to high levels of bacterial growth reached in the mutant (Fig. 1D). Interestingly, infection with the bacterial mutant strain $\mathrm{HrpC}$, affected in the type III secretion system (Deng et al. 1998), revealed no enhanced susceptibility in $\operatorname{scs} 9$ plants compared with Col-0 (Fig. 1E), while the nhoI Arabidopsis mutant, here used as a control for nonhost resistance, supported a 100-fold enhancement in bacterial growth (Fig. 1E). This observation suggested the enhanced susceptibility of scs 9 plants to DC3000 is host-specific and dependent on bacterial effectors. Potential changes in the susceptibility of $\operatorname{scs} 9$ plants to biotrophic pathogens were further investigated by inoculating plants with a virulent strain (WACO9) of the obligate oomycete Hyaloperonospora arabidopsidis. Disease severity was assessed at 7 days postinoculation in lactophenol trypan blue-stained leaves. The leaves were classified into four categories (I to IV) according to their degree of colonization by the oomycete (Fig. 1F; Supplementary Table S1). Both, Col-0 and $\operatorname{scs} 9$ plants exhibited a similar degree of colonization by the oomycete and showed no significant differences. This result indicates that SCS9 is not required for the immune response toward $H$. arabidopsidis and further substantiates its value in establishing an effective and specific immune response toward DC3000. In summary, these results point toward SCS9 as an important locus regulating plant resistance to DC3000 in Arabidopsis.

\section{scs 9 plants retain intact components} of innate and SA-mediated immunity.

The enhanced disease susceptibility of $\operatorname{scs} 9$ plants toward DC3000 might result from a lack of pathogen recognition or defects at any step downstream in the signaling pathway leading to activation of plant immune responses. Insights into 
the level at which $\operatorname{scs} 9$ might be operating were gained by studying major hallmarks of plant immune responses in search of defects that might explain the compromised mutant immunity. We first tested whether scs 9 plants might be affected in early recognition of pathogen-associated molecular patterns (PAMPs) by measuring PAMP-mediated deposition of the $\beta$-1,3-glucan cell-wall polymer callose. The degree of callose deposition induction in PAMP-treated leaves was monitored after staining with aniline blue, was examined by UV fluorescence microscopy (Fig. 2A), and was quantified by counting yellow pixels on digital images (Fig. 2B). The response to the bacterial flagellar peptide elicitor flg22 or to chitosan, which are unrelated PAMPs that induce callose deposition in Arabidopsis, was similar in $\operatorname{scs} 9$ and Col-0 seedlings (Fig. 2A and B). MYB51 gene expression (Clay et al. 2009) and mitogen-activated protein kinase (MAPK) activation (Asai et al. 2002; Bethke et al. 2009) constitute additional hallmarks for early PAMP-mediated immune signaling. Induction of MYB51 transcript accumulation, measured by reverse transcriptionquantitative polymerase chain reaction (RT-qPCR) at 5, 10, and 60 min after flg22 application, revealed no differences between Col-0 and $\operatorname{scs} 9$ plants (Fig. 2C). Early elicitation of MAPK activation following flg22 application was visualized by employing an antibody that recognized the phosphorylated residues within the MAPK activation loop. Western blot analysis of protein extracts derived from Col- 0 and $\operatorname{scs} 9$ plants revealed positive immunoreactive signals in the two polypeptides corresponding to MPK6 and MPK3 (Fig. 2D). Marked transient activation of both kinases occurred after flg22 application; activation was maximal at 5 to $10 \mathrm{~min}$ and then declined at $30 \mathrm{~min}$, remaining only partially active at $120 \mathrm{~min}$ after treatment. Results revealed no differences between $\operatorname{Col}-0$ and $\operatorname{scs} 9$ plants with respect to the pattern of MAPK activation (Fig. 2D). All these observations, therefore, indicated that the activation of the early stage of basal immunity was not affected in the $\operatorname{scs} 9$ mutant.

Fig. 1. Characterization of $s c s 9$ plants. A, Comparison of Col-0, $c s b 3$, and $\operatorname{cs} 33 \operatorname{scs} 9$ plants at 4 weeks after sowing. Note the stunted phenotype of the single $c s b 3$ mutant and the growth recovery conferred by the $\operatorname{scs} 9$ mutation in the double $\operatorname{csb} 3 \operatorname{scs} 9$ mutant. B, Comparative histochemical analysis of $\beta$-glucuronidase (GUS) activity in rosette leaves from a parental wild-type Col-0 plant carrying the pP69C:GUS transgene, which shows no constitutive expression of the transgene (left), from $c s b 3$ plants showing the constitutive activation of the transgene (center), and the reversion of this molecular phenotype in the double csb3 scs 9 mutant plant (right). C, Growth rates of virulent Pseudomonas syringae DC3000 in Col-0, npr1-1, $c s b 3, c s b 3 \operatorname{scs} 9$, and $\operatorname{scs} 9$ plants. Bacterial growth was measured at zero (white bars), 3 (gray bars), and 5 (black bars) days after spray inoculation with a bacterial inoculum at an optical density at $600 \mathrm{~nm}\left(\mathrm{OD}_{600}\right)$ of 0.1 . Error bars represent standard deviation (SD) $(n=12)$. Asterisks indicate statistical differences to Col- $0(P<0.05)$, using a Student's $t$ test. D, Representatives of DC3000-inoculated Col-0 and $\operatorname{scs} 9$ plants in which the $\operatorname{scs} 9$ mutant exhibits a distinguishable enhancement of chlorosis due to bacterial growth. E, Growth rate of Pseudomonas syringae pv. tomato $\mathrm{HrpC}$ in Col-0, scs 9 , and $n h o I$ plants. Bacterial growth was measured at 3 (black bars) and 5 (white bars) days after spray inoculation with a bacterial inoculum of $\mathrm{OD}_{600}=0.05$. Error bars represent $\mathrm{SD}(n=12)$. Asterisks indicate statistical differences to Col- $0(P<0.05)$, using a Student's $t$ test. F, Growth of Hyaloperonospora arabidopsidis in Col-0 and scs 9 plants. Three-week-old Arabidopsis seedlings were inoculated with a suspension of water containing $H$. arabidopsidis spores at a concentration of $10^{5}$ spores per milliliter. Around 200 leaves per genotype were stained with trypan blue solution, were analyzed, and were classified in four different categories, depending on the degree of the infection (pictures panel). Class $I=$ healthy leaves, class II = visible hyphal growth, class III = low degree of sporulation, and class IV = high degree of sporulation. The graph represents the distribution of leaves in each genotype. Differences in class distribution were statistically analyzed by a chi-square test. No statistically significant differences in the distribution of disease severity classes were observed between the two genotypes.
We next sought to demonstrate if, in a plant-DC3000 interaction, scs 9 plants might be defective in eliciting MAPK activation. Western blot analysis of protein extracts derived from Col-0, scs9, and $n p r 1$ plants, the latter used as a control of a mutant, also compromised immunity and revealed positive immunoreactive signals in the two polypeptides corresponding to MPK6 and MPK3, which were visible at 24 to $48 \mathrm{~h}$ postinoculation (hpi) with DC3000 (Fig. 2E). Results showed no notable differences among the three genotypes, suggesting $\operatorname{scs} 9$

A

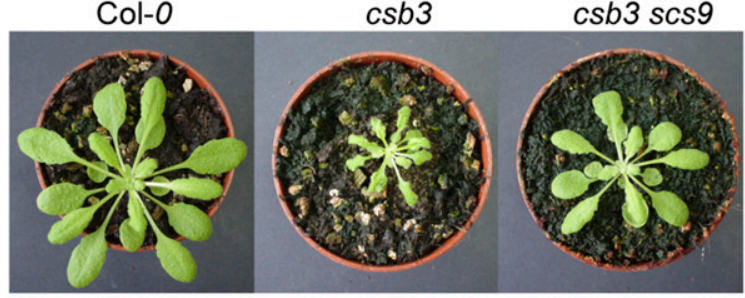

B
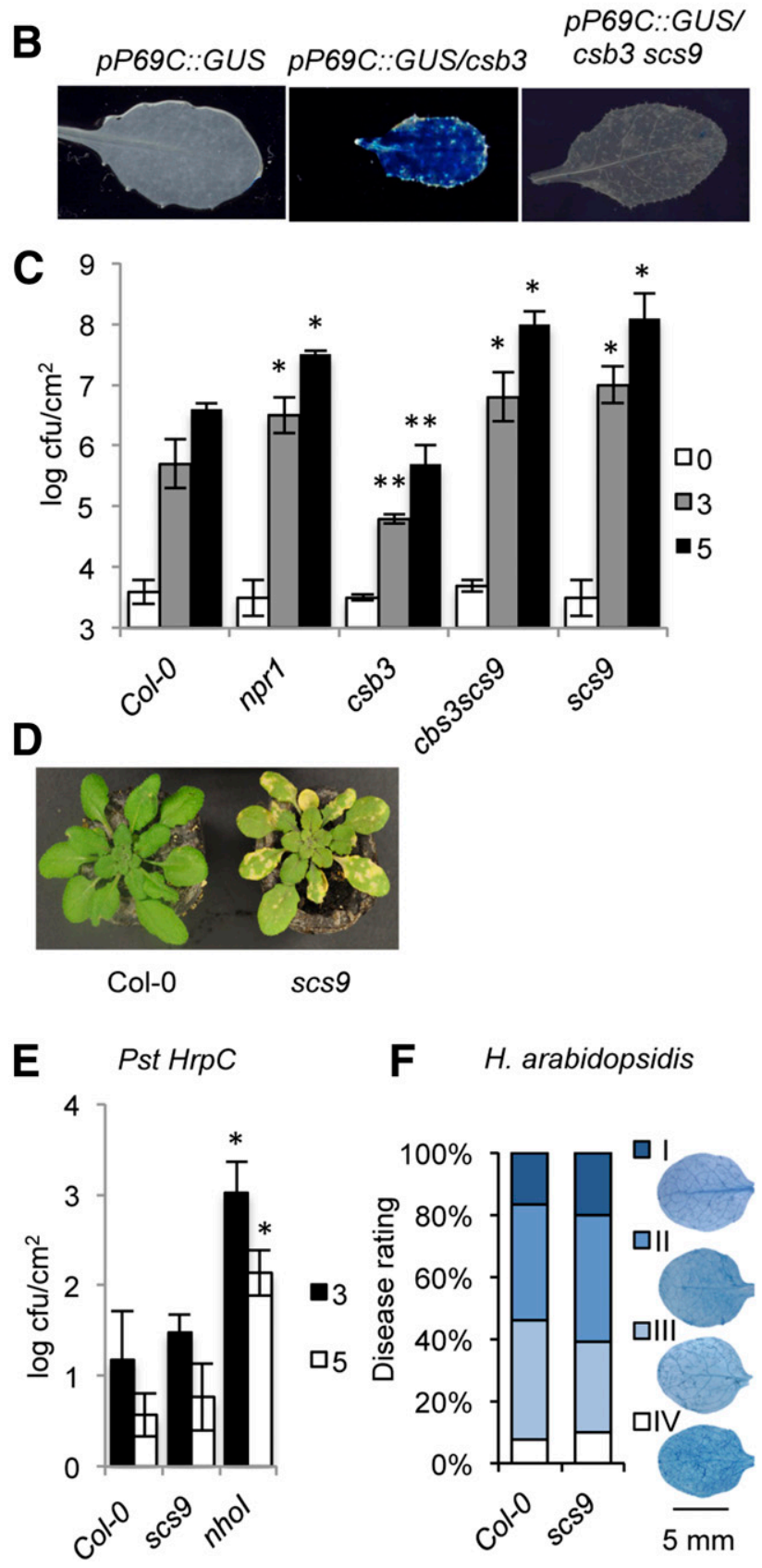

F H. arabidopsidis

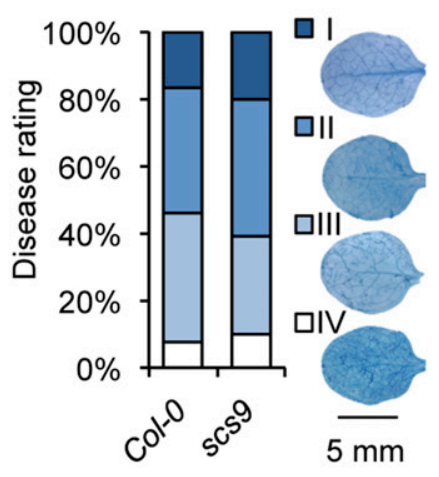


defects, as in $n p r l$, were positioned downstream of MAPK activation following pathogen perception.

Compromised synthesis or perception of SA is on the basis explaining the compromised immunity observed in different mutants (i.e., sid2 or $n p r 1$, respectively) (Cao et al. 1997; Nawrath and Métraux 1999). Consequently, we considered if scs 9 plants might carry defects in either SA biosynthesis, its accumulation, or both. Therefore, we conducted measurements to determine whether endogenous SA levels were affected in $\operatorname{scs} 9$ plants. Free SA and conjugated salicylate glucoside (SAG) concentrations were comparatively examined in leaf tissues from scs 9 and Col-0 plants following DC3000 inoculation (Fig. $2 \mathrm{~F})$. In $\operatorname{scs} 9$ plants, basal and induced amounts of free SA equaled the levels observed in Col-0 plants. SAG content was consistent with SA (Fig. 2F). This finding suggested that the increased susceptibility to DC3000 observed in $\operatorname{scs} 9$ plants was not due to defects in SA biosynthesis.

We subsequently examined whether SA perception was defective in $\operatorname{scs} 9$, as detected in the nprl mutant, in which compromised immunity was accompanied by the absence of

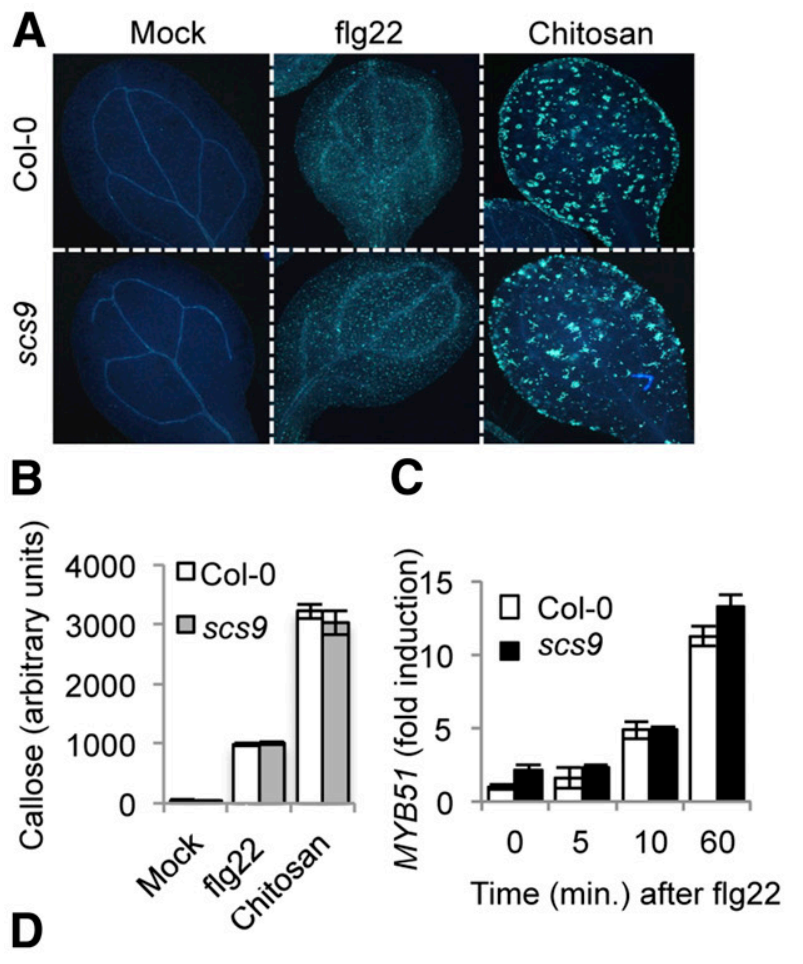

Col-0 $\operatorname{scs} 9$ Col-0 $\operatorname{scs} 9$
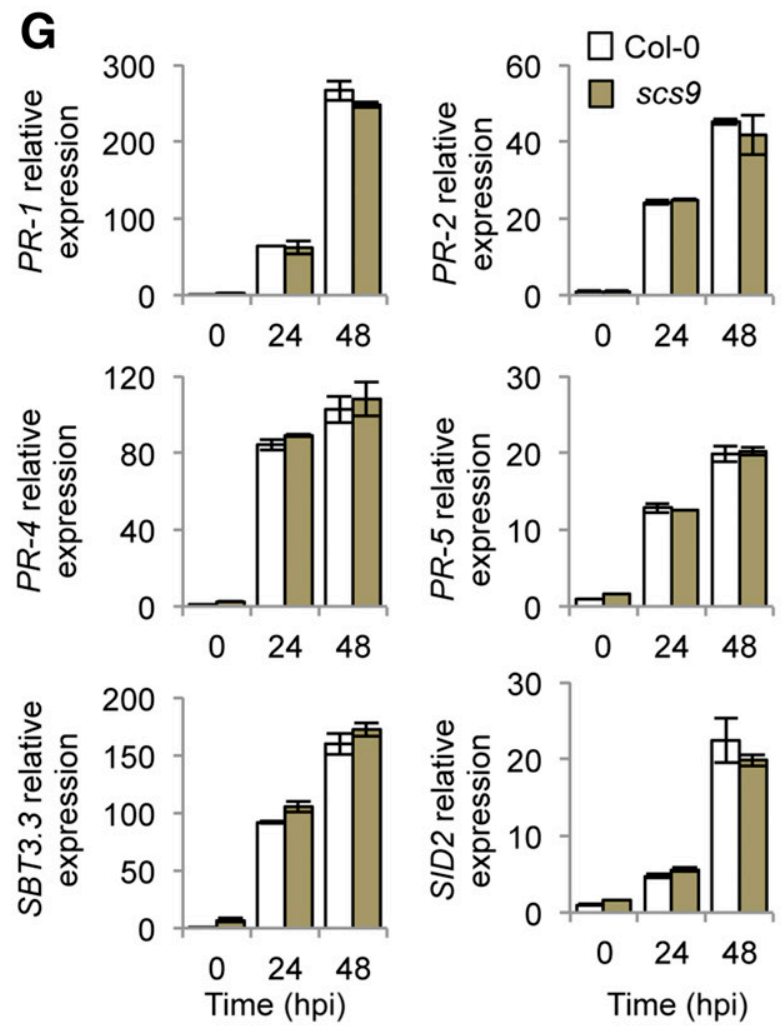

\footnotetext{
0 5' $10^{\prime} 30^{\prime} 0$ 5' 0 ' 30 ' 0 60' 120' 0 60' 120' Time (min.) after flg22

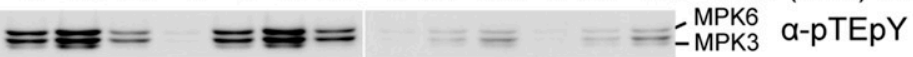

a-AtMAPK6

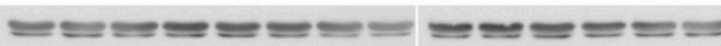

Ponceau-S
}

\section{E}
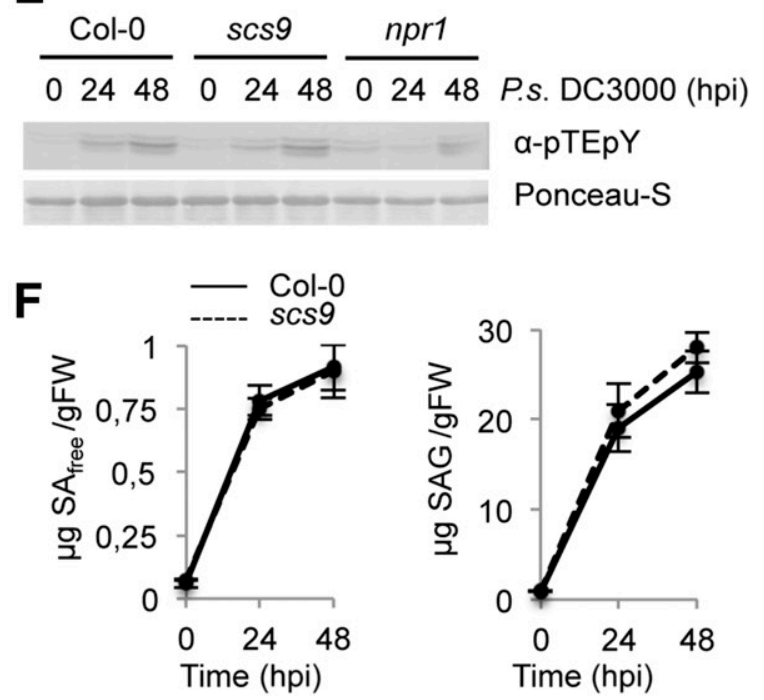

H

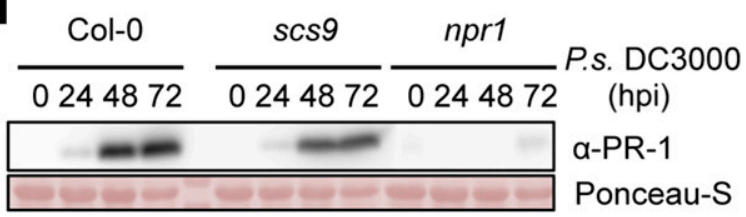

Col-0 scs9 Col-0 scs9 Col-0 scs 9

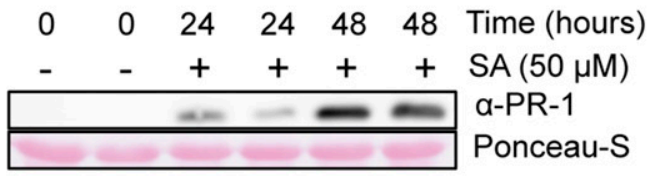


induced SA-responsive gene expression (Cao et al. 1997). Therefore, induced expression of several genes diagnostic of SA-mediated disease resistance was examined by RT-qPCR in $\operatorname{scs} 9$ and Col-0 plants following inoculation with DC3000. SAresponsive $P R-1, P R-2, P R-4, P R-5$, and SID2 gene expression profiles, or even the recently described SBT3.3 gene, which is pivotal in plant immunity (Ramírez et al. 2013), showed no differences in $\operatorname{scs} 9$ plants when compared with Col-0 at 0, 24, and 48 hpi (Fig. 2G). This indicated transcriptional reprogramming occurred in scs 9 plants following pathogen perception. This conclusion was further supported by Western blot analysis showing that induced accumulation of PR-1 protein after bacterial infection was similar in $\operatorname{scs} 9$ and Col-0 plants at 24 hpi; both genotypes showed higher accumulation of the protein at 48 and $72 \mathrm{hpi}$, with only a marginal difference in PR1 protein accumulation observed in the mutant (Fig. 2H; Supplementary Table S2). As expected, PR-1 accumulation was nearly absent in nprl plants, here used as a control (Fig. 2H). These results revealed differences between $\operatorname{scs} 9$ and nprl mutants and suggested the mutations are not allelic. Moreover, notable accumulation of PR-1 protein in response to exogenous SA application was observed in $\operatorname{scs} 9$ and Col-0 (Fig. 2I); only a very slight reduction in PR-1 accumulation could be observed in $\operatorname{scs} 9$ plants at $24 \mathrm{~h}$ after SA application further corroborating that $\operatorname{scs} 9$ plants show no severe defects in SA perception.

\section{SCS9 is At5g01230 and encodes a 2 ' $\boldsymbol{O} O$-ribose tRNA MTase.}

The $\operatorname{scs} 9$ position was mapped by outcrossing $\operatorname{scs} 9$ to Landsberg erecta (Ler) plants, and F2 plants were scored for cosegregation of enhanced susceptibility to DC3000 using simple sequence length polymorphisms (SSLPs). Thirty-five plants selected from a $\operatorname{Ler} \times \operatorname{scs} 9 \mathrm{~F}_{2}$ population were analyzed and $\operatorname{scs} 9$ was mapped to chromosome 5, in close proximity to the top telomeric region, and were linked to marker SGCSNP13418. We next collected $80 \mathrm{~F} 2$ individuals with the $\operatorname{scs} 9$ phenotype and isolated individual genomes, which were bulked and deepsequenced using the Illumina GAIIx platform. The resulting data were analyzed, using a bioinformatic pipeline devised by Austin et al. (2011), to identify a genomic region in which putative mutations of interest resided. Between the identified mapping intervals, we identified one nucleotide substitution corresponding to a $\mathrm{G}$ for $\mathrm{A}$ transition, as expected for a mutation caused by EMS mutagenesis. This resulted in a Ser for Asn substitution at position 194 of the protein encoded by the gene At5g01230 (Fig. 3A). Therefore, SCS9 should correspond to At5g01230.

We complemented the results of our cloning strategy by searching for existing SCS9 mutant alleles in T-DNA insertion mutant collections. One insertion mutant allele was detected, named here as $\operatorname{scs} 9-2$, which interrupted the third intron of the At5g01230 transcribed region (Fig. 3B). RT-qPCR analyses of SCS9 transcript levels in scs9-2 plants, compared with scs9-1 or
Col-0 plants, revealed the absence of transcript accumulation in the former mutant (Fig. 3C). The experiment also revealed the SCS9 gene was expressed in moderate levels in Col-0 and $\operatorname{scs} 9$ 1 and expression did not change following plant inoculation with DC3000 (Fig. 3C).

A comparison of disease resistance response to DC3000 between $\operatorname{scs} 9-1$ and $\operatorname{scs} 9-2$ plants showed both mutants were equally affected, indicating notable increased susceptibility. The heightened susceptibility was of a magnitude similar to that shown in $n p r l-1$ plants (Fig. 3D). The enhanced bacterial growth observed in the $\operatorname{scs} 9$ mutants was very robust and reproducible. Moreover, the enhanced bacterial growth observed in the $\operatorname{scs} 9-1$ and $\operatorname{scs} 9-2$ mutants and in $n p r l$ plants was accompanied by distinguishable symptoms of induced chlorosis. This visual phenotype was not dependent on the inoculation method used, since leaf inoculation with DC3000, by infiltration with a syringe or by spraying, induced enhanced chlorosis when compared with Col-0 (Supplementary Fig. S2). Moreover, when $\operatorname{scs} 9-1$ and $\operatorname{scs} 9-2$ seedlings were treated with flg22 $(1 \mu \mathrm{M})$, in a test for a microbe-associated molecular pattern-induced seedling-growth inhibition, none of the mutant alleles showed a decrease in the plant's ability to repress growth and development-related processes when compared with Col0 (Supplementary Fig. S3). This suggests that disruption of the growth to defense shift appears not to be the reason for the observed enhanced susceptibility of $\operatorname{scs} 9$ mutants to DC3000.

Next, we study the effect overexpression of SCS9 may have on disease resistance to DC3000. Agrobacterium-mediated plant transformation was used to introduce into scs9-1 (scs9OEXSCS9 plants) and Col-0 (wtOEXSCS9 plants) a cDNA corresponding to At5g01230, fused in-frame to green fluorescent protein (GFP) and under control of the constitutive 35S Cauliflower mosaic virus promoter. All 35S::SCS9 transgenic lines generated in a scs9-1 mutant background lost the characteristic enhanced disease susceptibility to DC3000 attributable to this mutation. Figure 3E compares bacterial growth for i) a transformed line in the $\operatorname{scs} 9-1$ genetic background (i.e., line 4.4), ii) a transgenic line in the Col0 background (i.e., line 7.4), and iii) both with respect to nontransformed scs $9-1$ and Col-0 plants. SCS9 expression restored enhanced disease susceptibility in $\operatorname{scs} 9-1$ plants to Col-0 levels, further supporting the conclusion that At5g01230 is SCS9.

An in silico genome search for homologous amino-acid sequences revealed the highest similarity of SCS9 was to bacterial FtsJ/RrmJ (Caldas et al. 2000), yeast Trm7p (Pintard et al. 2002), and human FTSJ1 (Bügl et al. 2000) proteins, all encoding 2'-O-ribose tRNA (MTases). Supplementary Figure S4 shows the conserved elements, including the predicted AdoMetand the ribose-binding sites of these four tRNA MTases and the conserved amino acids that conform the catalytic tetrad (K28, D126, K166, and E201) (Pósfai et al. 1989), which is a conserved feature in 2'-O-ribose MTases exhibiting the 'MTase fold' (Bujnicki and Rychlewski 2001; Kalhor and Clarke 2003).

Fig. 2. Characterization of the $\operatorname{scs} 9$ mutant. A, Callose deposition in Col-0 and scs 9 cotyledons following application of flg22 and chitosan. B, Callose accumulation was calculated as arbitrary units by quantifying the number of yellow pixels per million on digital micrographs of flg22- or chitosan-treated leaves at $24 \mathrm{~h}$. Bars represent mean \pm standard deviation (SD), $n=15$. C, Time-course reverse transcription-quantitative polymerase chain reaction (RT-qPCR) analysis showing MYB51 gene expression in Col-0 and scs 9 plants after application of flg22. Data represent mean \pm SD; $n=3$. D, Western blot with anti-pTEpY antibody of crude protein extracts derived from Col- 0 and $\operatorname{scs} 9$ plants at $0,5,10,30,60$, and 120 min after treatment with flg22. Below is shown a Western blot with an anti-AtMAPK6 antibody revealing that MPK6 protein accumulated to similar levels in all the samples. Staining of the nitrocellulose filter with Ponceau-S dye confirmed an equal protein loading. The experiments were repeated three times with similar results. E, Western blot with anti-pTEpY antibody from Col-0, scs 9 , and nprl-1 plants at 0,24 , and $48 \mathrm{~h}$ postinoculation (hpi) with Pseudomonas syringae DC3000. Equal protein loading was verified by staining of the nitrocellulose filter with Ponceau-S dye. The experiments were repeated three times with similar results. F, Free salicylic acid (SA) and conjugated salicylate glucoside (SAG) accumulation in Col-0 and $\operatorname{scs} 9$ mutant at 0, 24, and 48 hpi with DC3000. Data represent the mean \pm SD; $n=3$ replicates. G, Timecourse RT-qPCR analysis showing $P R-1, P R-2, P R-4, P R-5, S B T 3.3$, and SID2 gene expression in Col-0 and scs 9 plants after infection with DC3000. Data represent mean $\pm \mathrm{SD} ; n=3$. H, Western blots with anti-PR-1 antibody showing inhibition of PR-1 protein accumulation in $n p r l$ compared with Col- 0 or in $s c s 9$ mutant plants following inoculation with DC3000 and I, induced accumulation of PR-1 protein following application of SA (50 $\mu \mathrm{M})$ in Col-0 and scs 9 plants. Experiments were repeated three times with similar results. 
Arabidopsis SCS9 and the orthologous MTases from bacteria, yeast, and humans exhibited strong conservation to the predicted active site and its neighborhood, suggesting similarity to their target RNA.

Pintard et al. (2002) proposed a tRNA substrate interaction model and predicted the yeast Trm7 protein solvent-exposed side chain of S197 and R194 residues, equivalent to S199 and R196 in the Arabidopsis SCS9 protein, interacts specifically with the phosphate group of the methylated nucleotide. The $\operatorname{scs} 9-1$ mutation is located where the conserved $\mathrm{S} 194$ is

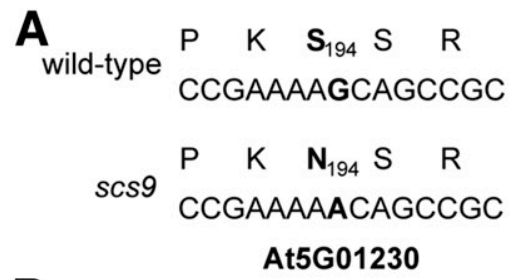

B
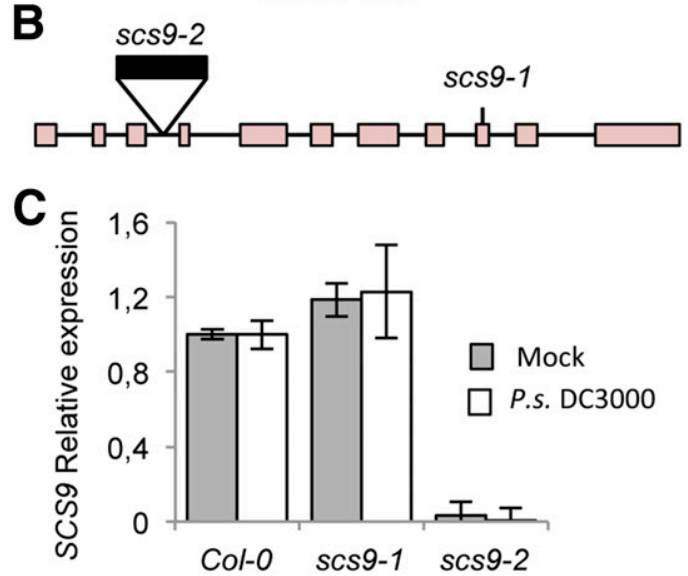

D
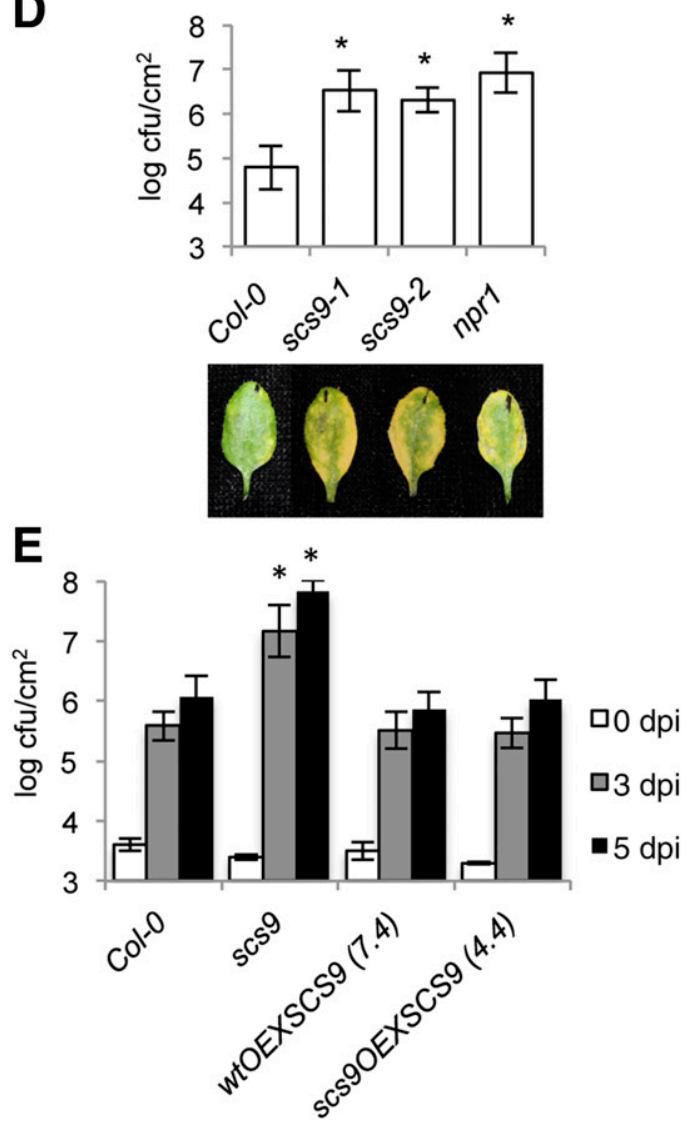

substituted by N194 and lies very proximal to the mentioned residues and the catalytically important E201 tetrad residue. Therefore, it is very likely the $\operatorname{scs} 9-1$ loss-of-function might be due to a lack of enzymatic activity, which would reveal the importance of the $\mathrm{S} 194$ residue for $2^{\prime}$ - $O$-tRNA MTases.

\section{SCS9 complements the yeast $t r m 7 \Delta$ mutant.}

Similarity between Arabidopsis SCS9 and the bona fide S. cerevisiae Trm7p tRNA MTase amino-acid sequences led us to investigate whether SCS9 might functionally mimic Trm7p. The deleted $S$. cerevisiae trm $7 \Delta$ strain phenotype is characterized by slow growth compared with the wild-type strain (Pintard et al. 2002). Therefore, to examine whether the SCS9 gene provided the functions of the fission yeast TRM7 gene, we attempted complementation of a $\operatorname{trm} 7 \Delta$ mutant strain. Unlike the vector alone (Fig. 4A), the yeast-Escherichia coli shuttle vector carrying the SCS9 cDNA complemented the trm7 1 mutant slowgrowth phenotype (Fig. 4A). We therefore concluded the SCS9 gene is the Arabidopsis functional homolog of the TRM7 gene.

\section{scs9 mutant is hypersensitive}

to paromomycin and rose bengal.

The yeast $\operatorname{trm} 7 \Delta$ strain has been described to be highly sensitive to the antibiotic paromomycin (Pintard et al. 2002), which impairs translation by increasing codon misreading in prokaryotes and eukaryotes (Chernoff et al. 1994). Therefore, we hypothesized if SCS9 and Trm7p are functional homologs, then a $\operatorname{scs} 9$ Arabidopsis strain might also show enhanced sensitivity to paromomycin. The $\operatorname{scs} 9-1$ strain carries the kanamycinresistance gene as a selectable marker, which might interfere with the antibiotic sensitivity assay; therefore, we employed the scs 9-2 allele that, instead, carries resistance to the herbicide BASTA as the selectable marker. Col-0 seedlings were moderately sensitive to 0.005 to $0.01 \%$ paromomycin, which partially slowed plant growth and elicited moderate anthocyanin deposition in leaves, presumably as a consequence of antibiotic imposed stress (Fig. 4B to D). Interestingly, the $\operatorname{scs} 9-2$ mutant showed enhanced sensitivity to the antibiotic, as indicated from the decreased seedling growth and a concurrent increase in anthocyanin deposition in leaves of paromomycin-treated $\operatorname{scs} 9-2$ plants (Fig. 4B to D). These effects were more evident at $14 \mu \mathrm{M}$ $(0.01 \% \mathrm{wt} / \mathrm{vol})$ compared with $7 \mu \mathrm{M}(0.005 \% \mathrm{wt} / \mathrm{vol})$ antibiotic concentration, which also maximized the differences between Col-0 and $\operatorname{scs} 9-2$ seedling growth. Therefore, consistent with the yeast $\operatorname{trm} 7 \Delta$ mutant, the Arabidopsis scs9-2 mutant was also highly sensitive to paromomycin.

Fig. 3. $\operatorname{scs} 9$ is a mutant allele of At5g01230. A, SCS9 corresponds to At5g01230. The G nucleotide residue mutated to an A nucleotide in the $\operatorname{scs} 9$ allele is indicated in bold letters. The deduced amino-acid sequences are indicated above each nucleotide sequence, and the deduced amino-acid change (S194 to N194) is shown in bold. B, Intron-exon arrangement in At5g01230. Exons are shown as boxes. The distances are only approximate. Position of a T-DNA insertion in the third intron (indicated by a black box) and localization of the ethyl methanesulfonate-induced mutation in the ninth exon, rendering $\operatorname{scs} 9-2$ and $\operatorname{scs} 9-1$ mutant alleles, are indicated. $\mathbf{C}$, Reverse transcription-quantitative polymerase chain reaction (RT-qPCR) analysis of the SCS9 transcript accumulation levels in mock- and Pseudomonas syringae DC3000-inoculated Col-0, scs9-1, and scs9-2 plants at 3 days postinoculation (dpi). Data represent the mean \pm standard deviation (SD), $n=3$. D, Comparative growth of DC3000 in Col-0, scs9-1, scs9-2, and $n p r l$ plants 3 dpi. Error bars represent SD $(n=12)$. Below are representatives of inoculated leaves of the indicated genotypes. E, SCS9 overexpression complements the scs $9-1$ mutant, and bacterial growth titers reach Col-0 levels. Plants of the indicated genotype were inoculated with DC3000. Bacterial growth was measured 0 (white bars), 3 (gray bars), and 5 (black bars) dpi. Error bars represent SD $(n=12)$. Asterisks indicate statistical differences compared with Col-0 $(P<0.05)$, analyzed using a Student's $t$ test. 
Moreover, Khoury et al. (2008) reported the $\operatorname{trm} 7 \Delta$ strain was more sensitive to oxidative stress and exhibited enhanced growth inhibition in the presence of reactive oxygen species (ROS) (i.e., $\mathrm{H}_{2} \mathrm{O}_{2}$ ). Therefore, we examined if the $\operatorname{scs} 9$ mutant became similarly more sensitive to oxidative stress. We treated Arabidopsis seedlings with a synthetic dye, rose bengal, which results in conditional ROS formation in the presence of light (Różanowska et al. 1995), and we compared the responsiveness of Col-0 and scs $9-2$ seedlings to this agent. Col-0 seedlings grown in petri dishes showed sensitivity after 5 days of exposure to $2 \mu \mathrm{M}$ rose bengal, which elicited partial chlorosis in leaves, reduction in root growth, and inhibition of lateral root formation (Fig. 4E). scs9-2 mutant seedlings exhibited increased sensitivity to the dye, which became bleached instead of chlorotic, and seedlings eventually died after 5 days of exposure (Fig. 4E). Therefore, both yeast trm $7 \Delta$ and Arabidopsis $\operatorname{scs} 9$ mutants displayed hypersensitive responses to this type of ROS-derived stress condition. In contrast, $\operatorname{scs} 9-1, \operatorname{scs} 9-2$, and Col-0 seedlings are equally sensitive to salt-derived stress, as recorded in seedling growth inhibition and germination assays performed with increasing concentrations (i.e., 0, 50, 100, and $200 \mathrm{mM}$ ) of $\mathrm{NaCl}$ (Supplementary Fig. S5). These differences might indicate a selectivity for this specific tRNA $2^{\prime}$ - $O$-ribose MTase in mediating adaptation to specific stressing situations.

\section{SCS9 localizes to the nuclei, including the nucleolus.}

Although tRNA molecules are transcribed in the nucleus, they can function in different subcellular compartments, emphasizing the importance of an appropriate distribution and localization of tRNA modifying enzymes. Moreover, mature tRNA move from the cytoplasm to the nucleus via retrograde tRNA nuclear import, a process proposed to function as a pathway that monitors end processing of pretRNAs (Kramer and Hopper 2013). Our objective was to identify the cellular compartment in which SCS9 exerted its function as a potential tRNA modifier. We therefore used a SCS9 tagged to green fluorescent protein (GFP) or mCherry protein, which was functional in complementing the $\operatorname{scs} 9$ phenotype (Fig. 3E), and performed colocalization studies using confocal microscopy. The free GFP or specific subnuclear compartment marker proteins were used upon transient agroinfiltration of Nicotiana benthaminana leaves with Agrobacterium strains carrying each corresponding gene construct. eIF4A, a RNA helicase component of the exon junction complex (eiF4A-mRFP) and the histone $\mathrm{H} 3$ variant cenH3 (CENH3-mRFP) colocalized with SCS9-mCherry in the nucleolus and nucleoplasm (Fig. 5; panels iv and v), indicating SCS9 partial enrichment or preference for the nucleolus. Coilin (Coilin-mRFP) and U2b" (U2b"'-GFP), two Cajal body markers and other nuclear bodies

Fig. 4. Complementation of the Saccharomyces cerevisiae trm $7 \Delta$ strain with Arabidopsis SCS9 cDNA and hypersensitivity of $\operatorname{scs} 9-2$ seedlings to paromomycin and rose bengal. A, Comparative growth curves of various $S$. cerevisiae strains grown in yeast potato dextrose at $30^{\circ} \mathrm{C}$. Open squares represent wild-type strains (BMA64) transformed with plasmid p423GAL1 alone (WT ccdB) or using a plasmid carrying an Arabidopsis SCS9 cDNA (WT SCS9). Filled circles represent the trm7 $\Delta$ strain transformed with the plasmid alone (trm $7 c c d B$ ), or with the plasmid carrying the Arabidopsis SCS9 cDNA (trm7 SCS9). B, Col-0 and scs9-2 seedling growth in mass spectrometry (MS) plates alone or in the presence of $0.005 \%$ (wt/wt) and $0.01 \%(\mathrm{wt} / \mathrm{wt}$ ) of paromomycin. C, Quantitative analysis of paromomycin effects on growth retardation in Col-0 and scs 9-2 seedlings measured as reduction in fresh weight (FW). D, Quantitative analysis of anthocyanins accumulation in Col-0 and scs $9-2$ seedlings treated with paromomycin at the indicated concentrations. E, Differential effects of rose bengal on growth inhibition of Col-0 and scs $9-2$ seedlings grown in MS plates. often associated with the nucleolus, also confirmed SCS9 localization in the nucleus and its preference for the nucleolus but not for any type of nuclear bodies (Fig. 5; panels ii and iii). Finally, free GFP, which accumulated in the cytoplasm and nucleus but was excluded from the nucleolus, allowed a clear identification of SCS9-mCherry in the nucleolus (Fig. 5; panel i). Therefore, we concluded that SCS9 is preferentially localized in the nucleus and exhibited preference for the nucleolus. These results suggested modifications of tRNA molecules,

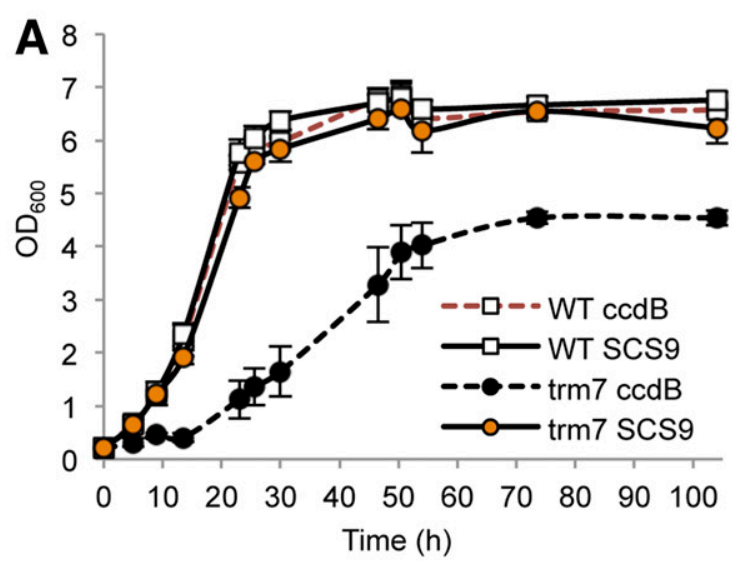

B
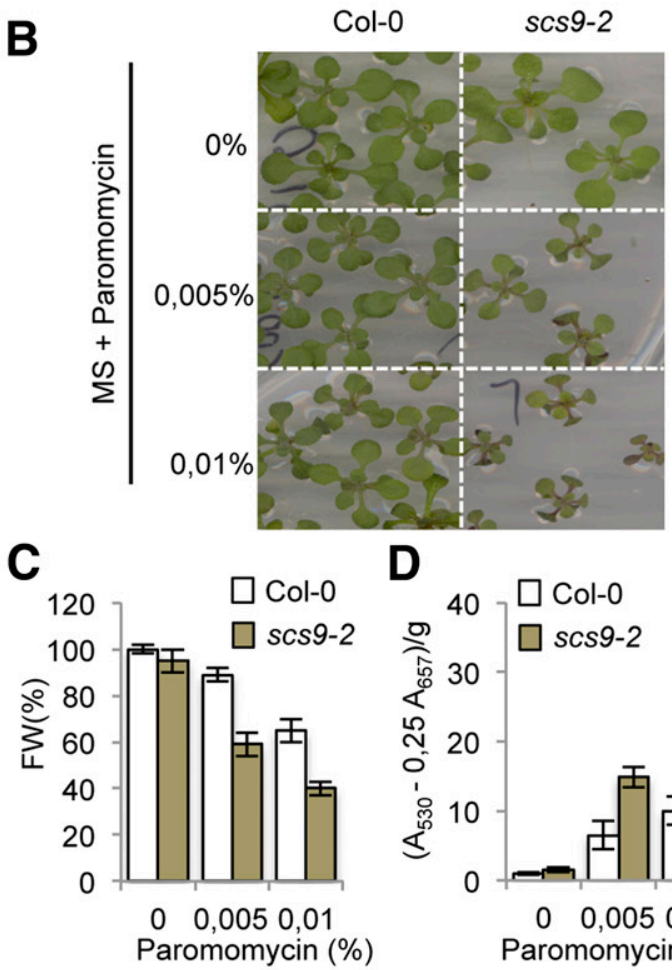

D

E
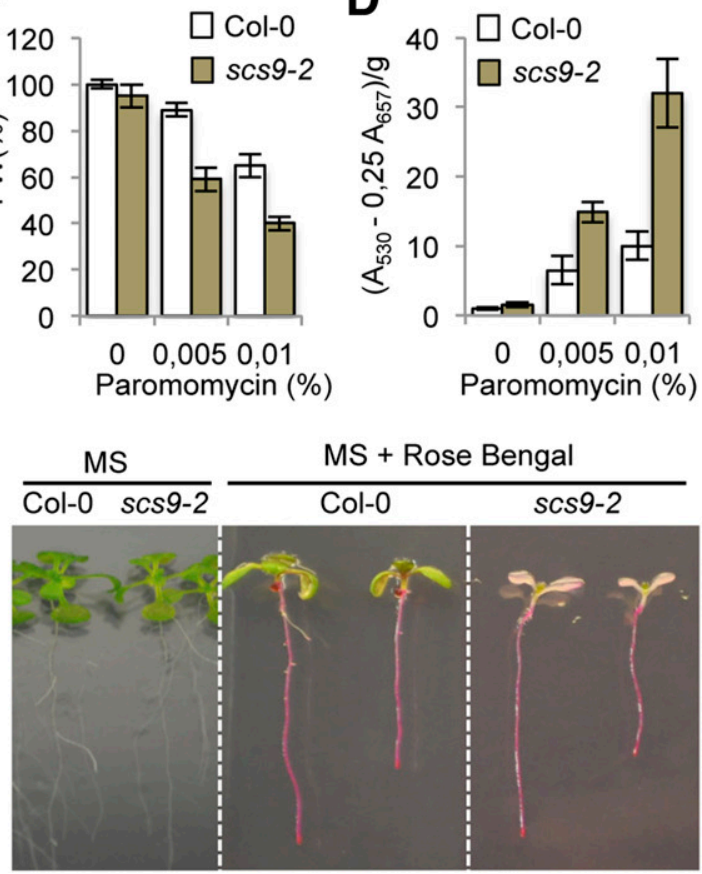
directed by the MTase enzyme, most likely occur in the nucleus and not the cytoplasm.

\section{SCS9 is required}

\section{for tRNA $2^{\prime}$ - $O$-ribose methylation in vivo.}

Based on the close structural similarities between SCS9 and Trm7p, FtsJ/RrmJ, and FTSJ MTases, we tested whether SCS9 was similarly involved in $2^{\prime}$-O-ribose methylation of nucleotides in the Arabidopsis tRNAs anticodon loop. Positions 32 and 34 in the anticodon loop (Fig. 6A) were identified as concurrent targets of Trm7p-like MTases (Pintard et al. 2002). Modified nucleotides corresponding to Arabidopsis tRNAs potentially altered due to defects in SCS9 were identified by comparing Col-0 modifications with those that commonly differed in $\operatorname{scs} 9-1$ and $\operatorname{scs} 9-2$ mutants. We used young seedlings as a source of plant material, which, according to our previous experiences, show higher abundance of RNA. tRNA isolation, degradation, and subsequent high-pressure liquid chromatography (HPLC) analysis of separated nucleosides were

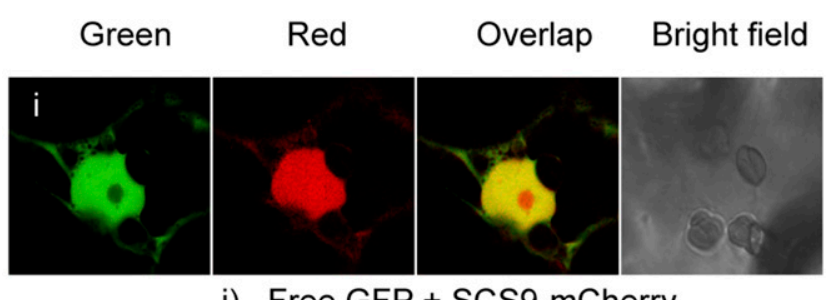

i) Free GFP + SCS9-mCherry

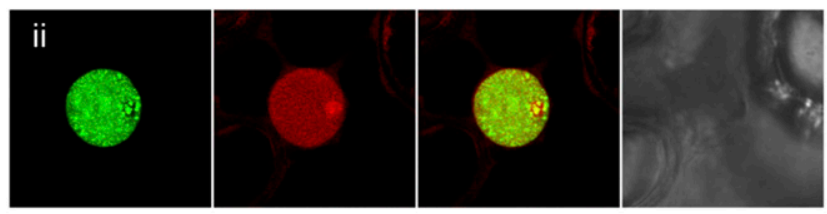

ii) U2b"-GFP + SCS9-mCherry

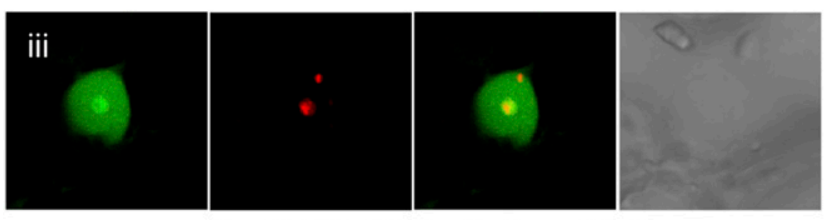

iii) SCS9-GFP +Coilin-mRFP

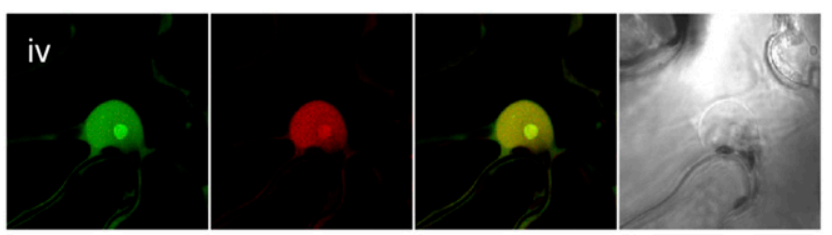

iv) SCS9-GFP + elF4A-mRFP

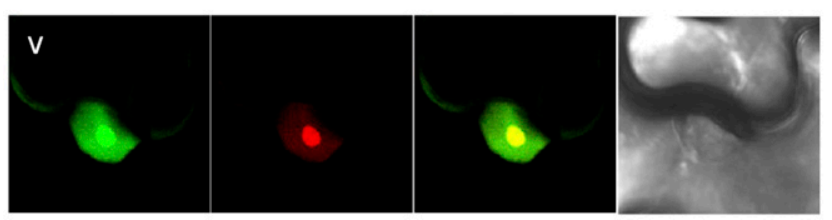

v) SCS9-GFP + CENH3-mRFP

Fig. 5. SCS9 nuclear localization. Colocalization patterns of SCS9, fused to either green fluorescent protein (GFP) or mCherry, with free GFP (panel i) and several different nuclear tagged proteins, including U2b" (panel ii), Coilin (panel iii), eIF4A (panel iv), and CENH3 (panel v) in epidermal cells from Nicotiana benthamiana transfected with each respective pair of constructs, evaluated by confocal microscopy. performed as previously described (Chen et al. 2010); elution time, and spectrum of each peak were used to identify different modified nucleosides. Characteristic chromatograms of Col-0, $\operatorname{scs} 9-1$, and $\operatorname{scs} 9-2$ plants are shown in Supplementary Figure S6. Twenty-one major modified nucleosides were detected in the three genetic backgrounds, which all fit into previously identified modifications in Arabidopsis (Chen et al. 2010). Notably, HPLC chromatogram comparison between Col-0 and the two mutants revealed $\operatorname{scs} 9-1$ and $\operatorname{scs} 9-2$ plants carried identical conspicuous diminutions in $2^{\prime}-O$-methylated cytidine $(\mathrm{Cm})$ content when compared with other invariant modified nucleosides such as 7-methyl guanosine $\left(\mathrm{m}^{7} \mathrm{G}\right)$ or 1-methyl guanosine $\left(\mathrm{m}^{1} \mathrm{G}\right)$. Cm-modified nucleosides showed $70 \%$ reduction in $\operatorname{scs} 9-1$ and $\operatorname{scs} 9-2$ plants compared with Col-0 plants (Fig. 6B). In addition, $2^{\prime}-O$-methylated uridine (Um) content showed a $30 \%$ reduction in both mutants (Fig. 6B). However, in $\operatorname{scs} 9-1$ and $\operatorname{scs} 9-2$ mutants, the decrease in $2^{\prime}-O$-methylated guanosine $(\mathrm{Gm})$ was consistently much less, at only 10 to $15 \%$ reduction (Fig. 6B). More accurate and straightforward determination of $2^{\prime}-O$-methylated cytosine $(\mathrm{Cm})$ content by liquid chromatography-mass spectrometry (LC-MS) confirmed the notable reduction observed by HPLC analysis in $s c s 9-1$ and scs9-2 plants when compared with Col-0 plants (Fig. 6C). Interestingly, following DC3000 inoculation, Col-0 plants showed a moderate but progressive accumulation of $\mathrm{Cm}$ with a statistically significant $60 \%$ enhancement occurring at 72 and 120 h.p.i when compared with its control at 0 hpi (Fig. 6C). Conversely, scs $9-1$ and $s c s 9-2$ plants were impaired in this pathogen-mediated enhancement of $\mathrm{Cm}$ (Fig. 6C). For Um nucleoside modification (Fig. 6C), and to a lesser extent also for Gm (Supplementary Fig. S7), LC-MS quantification revealed similar enhanced accumulation in Col-0 at 72 to 120 hpi. These enhancements were not observed to occur in $\operatorname{scs} 9-1$ and $\operatorname{scs} 9-2$ plants (Fig. 6C).

The three nucleoside modifications are highly conserved modifications in the tRNA anticodon loop, Cm occurring at positions 32 and 34, $\mathrm{Um}$ at position 32, and $\mathrm{Gm}$ at position 34 (Fig. 6A). Positions 32 and 34 are known targets of Trm7p-like MTase, it is therefore likely that SCS9 is similarly required for $2^{\prime}$-O-ribose methylation at position 32 and 34 . In yeast, Wilkinson et al. reported cytosine and uracil nucleotides at position 4 of tRNAs (Fig. 6A) were also 2'-O-methylated (Wilkinson et al. 2007); however at this position ribose methylation was Trm7p-independent, and instead required the Trm13p methyltransferase. It remains unknown whether $\mathrm{Um}$ and $\mathrm{Cm}$ at position 4 occurs in plants, and if a Trm13-like enzyme regulates these specific modifications.

The $2^{\prime}$ - $O$-ribose modifications at positions 32 and 34 of the anticodon loop revealed by our study occurred in tRNA ${ }^{\text {Phe(GmAA) }}$, tRNA $^{\operatorname{Trp}(\mathrm{CmCA})}$ and tRNA ${ }^{\mathrm{Leu}(\mathrm{UmAA})}$ (El Yacoubi et al. 2012; Pintard et al. 2002). Reduced $2^{\prime}$ - $O$-methylated nucleoside accumulation observed in $\operatorname{scs} 9-1$ and $\operatorname{scs} 9-2$ mutants might result from reduced accumulation of the respective tRNA precursors. We therefore performed Northern blots of small RNAs extracted from Col-0 and the two scs 9 mutants, and hybridized the filters with radiolabeled probes specific for each of the three indicated tRNAs. The nonrelated tRNA ${ }^{\text {Gln }}$ was used as an internal control. Noticeable changes in RNA accumulation were not observed in any genetic backgrounds for any tRNAs examined (Fig. 6D). In addition, alterations in tRNA content were not detected following DC3000 inoculation. However, at this stage we cannot disregard the possibility that other functional aspects of tRNA metabolism, like those related to the relative level of charged and uncharged tRNAs may become altered in the $\operatorname{scs} 9$ mutants.

Therefore, the marked reduction in $\mathrm{Cm}$ and Um accumulation, and to a minor extent in $\mathrm{Gm}$, common to the $\operatorname{scs} 9$ mutants, 
is a consequence of $2^{\prime}-O$-methyl transferase loss-of-function, and which appeared to have no effect on transcript levels of corresponding tRNAs.

\section{DISCUSSION}

In the present report, we employed a variety of approaches to define a relationship between specific tRNA modifications at the anticodon loop, and the execution of an effective immune response toward DC3000 in Arabidopsis. Identification of the $s c s 9$ mutant, which suppressed the previously described $c s b 3$ associated enhanced disease resistance phenotype, indicated that SCS9 was pivotal for plant resistance toward this pathogen. Moreover, the observation that $\operatorname{scs} 9$ plants allow a similar growth of the biotrophic pathogen $H$. arabidopsidis indicates that SCS9 is required to mount an effective resistance specifically against $P$. $s$. DC3000. In addition, $\operatorname{scs} 9$ shows a wild-typelike response to this pathogen when its Type III secretion system is removed, suggesting that the enhanced susceptibility of $\operatorname{ses} 9$ plants to DC3000 is dependent on bacterial effectors and thus related to effector-triggered immunity (ETI). In $\operatorname{scs} 9$ plants the molecular hallmarks associated with activation of immune responses were properly activated; however, despite these measures scs 9 plants were unable to fend off DC3000. Therefore $\operatorname{scs} 9$ plants posses a critical distinction that differentiates $\operatorname{scs} 9$ from other mutant plants with a similar compromised immunity. NPR1 is the central regulator of SAmediated plant immunity toward DC3000 (Yan and Dong 2014), and therefore the nprl mutant is unable to resist DC3000 infections. However, at variance with the nprl mutant, scs 9 plants are not compromised in SA perception and neither on its accumulation. This makes a clear distinction between $n p r l$ and $s c s 9$ plants, suggesting that the defect(s) in scs 9 plant resistance operate downstream or distant to the point of $n p r l$ failed control.

Map-based cloning and sequence analysis revealed SCS9 is homologous to yeast Trm7p (Pintard et al. 2002), human FTSJ1 protein (Bügl et al. 2000), and bacterial FtsJ/RrmJ proteins (Caldas et al. 2000), encoding 2'-O-ribose tRNA MTases. These enzymes exhibited the common "MTase fold" (Bujnicki and Rychlewski 2001), suggesting their biological target tRNA must be similar. In fact, SCS9 provided the fission yeast Trm7p functions, demonstrated by effective complementation of a trm7 7 mutant using Arabidopsis SCS9 cDNA, and providing unequivocal evidence the SCS9 gene is the Arabidopsis functional homolog of the TRM7 gene. The mechanistic relationship between Trm7p and SCS9 was further established by the observation that loss of either protein makes corresponding trm $7 \Delta$ and $\operatorname{scs} 9$ mutant cells sensitive to oxidative stress and also sensitive to paromomycin, an antibiotic that impairs translation by increasing codon misreading (Chernoff et al. 1994). However, this enhanced sensitivity of the $\operatorname{scs} 9$ mutants is not manifested when other type of abiotic stress, such as saline stress, is imposed. These results thus give support to the importance of this specific tRNA $2^{\prime}$-O-ribose MTase for plants to selectively cope with only certain types of stress and adds to the growing body of evidences indicating that other tRNA nucleoside modification genes are pivotal to cope with imposed stress conditions (Burgess et al. 2015; David et al. 2017; Huang and Hopper 2016; Wang et al. 2017; Zhou et al. 2013). Moreover, consistent with trm7 $\Delta$, Arabidopsis scs 9 plants were defective in the $2^{\prime}$ - $O$-ribose methylation of nucleotides 32 and 34 of the tRNAs anticodon loop. The marked reduction in $\mathrm{Cm}$ content and, to a lesser degree, in Um and Gm at nucleotides 32 and 34, all of them targets of yeast Trm7p MTase (Pintard et al. 2002), reinforced our conclusion that Arabidopsis SCS9 and yeast Trm7p MTases are homologous. Furthermore, the observation that $\mathrm{Cm}$ content and, to a similar extent, also Um content enhance upon infection of Col-0 plants with DC3000 and that their deficiency correlates with a defective immune response gives relevance to SCS9 as a new component regulating effectiveness of plant resistance against DC3000. Our observation on the importance of tRNA modification in biotic stress response thus adds to the observation of Pajerowska-Mukhtar et al. (2012), who reported that the levels of uncharged tRNA corresponding to phenylalanine were shown to rapidly and transitorily increase in response to pathogen attack to allow translation of TBF1, a key defense regulator important to mediated SA-mediated immunity (Xu et al. 2017a). Even though this latter study focused on a different regulatory step of translation in plant immunity, the evidences also points toward the importance of tRNA metabolism as a point of control regulating biotic stress adaptation.

Then, how can a defect in Cm at specific tRNA wobble positions interfere with plant resistance while maintaining an intact upstream signal transduction pathway normally required for mounting an efficient plant immune response? We speculate that, following DC3000 inoculation, reprogramming of specific tRNA wobble modifications led to selective translation of mitochondrial RNA (mRNA) species enriched with the cognate codon, in a manner similar to that described in other eukaryotes (Chan et al. 2010; Wang and He 2014). The absence of accurate modifications at the wobble position in specific tRNAs would drive inefficient translation or generate misreading of specific proteins, which would be vital for an effector-triggered immune response. This translational control is congruent with SCS9 operating downstream of NPR1 and of its subordinated transcriptional reprograming during the defense response. Moreover, these observations represent an additional layer of complexity in the control of the global translational reprogramming that occurs during induction of resistance in plants (Xu et al. 2017b) and gives further support to the existence of mechanisms in which tRNA-derived nucleoside modifications control translation of proteins under stress (Chan et al. 2012; Dedon and Begley 2014; Gu et al. 2014). However, it is important to note $\operatorname{scs} 9$ mutants carried no gross alterations at the protein synthesis and accumulation levels, as deduced from the normal protein pattern observed in sodium dodecyl sulfate (SDS) polyacrylamide gels or from the normal accumulation of host-induced pathogenesis-related proteins (e.g., PR-1) interpreted from Western blot analysis. This favors a hypothesis in which defects derived from $\operatorname{scs} 9$ mutations would very likely elicit impacts at the proteome level in a very specific manner, presumably affecting only translation in a selective group of proteins with a pivotal role for effective execution of the immune response program. The literature provides some evidence for specific tRNA modification-dependent translation control during stress response in higher eukaryotes and, particularly, to oxidative stress. For example, Kalhor and Clarke (2003) showed that yeast Trm9 catalyzed methyl esterification leads to conversion of uracil into $\mathrm{mcm}^{5} \mathrm{U}$ at the wobble position of tRNA $^{\operatorname{Arg}(\underline{U} C U)}$ and tRNA ${ }^{\text {Glu(UUUC) }}$. This process enhanced binding of the anticodon and, therefore, facilitated translation of AGA- and GAA-rich transcripts, which functionally maps to processes of stress signaling to fend off deleterious effects of ionic radiation on DNA damage (Begley et al. 2007). Similarly, Trm4, which catalyzes $\mathrm{m}^{5} \mathrm{C}$ at the wobble position of tRNA ${ }^{\text {Leu(CAA) }}$, enhanced translation efficiency of mRNAs enriched in the UUG codon recognized by this tRNA, and loss of Trm4 caused hypersensitivity to the cytotoxic effects of $\mathrm{H}_{2} \mathrm{O}_{2}$ (Jackman et al. 2003), a phenotype also observed to occur in the trm $4 b$ mutant defective in a tRNA MTase that renders 5-methylcytosine $\left(\mathrm{m}^{5} \mathrm{C}\right)$ (David et al. 2017). One of these UUG-biased mRNAs whose translation is controlled 
by Trm4 is the ribosomal protein Rpl22A, whose increase in translation is required to cope with oxidative stress (Chan et al. 2012). This observation adds to a growing acceptance of the role for functional diversity in ribosome composition and for ribosomes in selective translation of proteins (Mauro and Edelman 2007). Interestingly, this reprograming of the translation machinery reconciles with the proposed generation of 'immunoribosomes' as a subset of T-cell ribosomes, responsible for translating peptides involved in antigen presentation (Yewdell et al. 1996). These are only examples illustrating toward the enormous complexity underlying translation control mechanisms and our limited understanding, particularly in plants. Therefore, the variety of tRNA modifications, its complex regulation, and the established biases in gene codon distributions point toward the existence of mechanisms evolved to fine tune the translational response to any cell stimulus, such as those suggested to regulate immunity by SCS9.
A

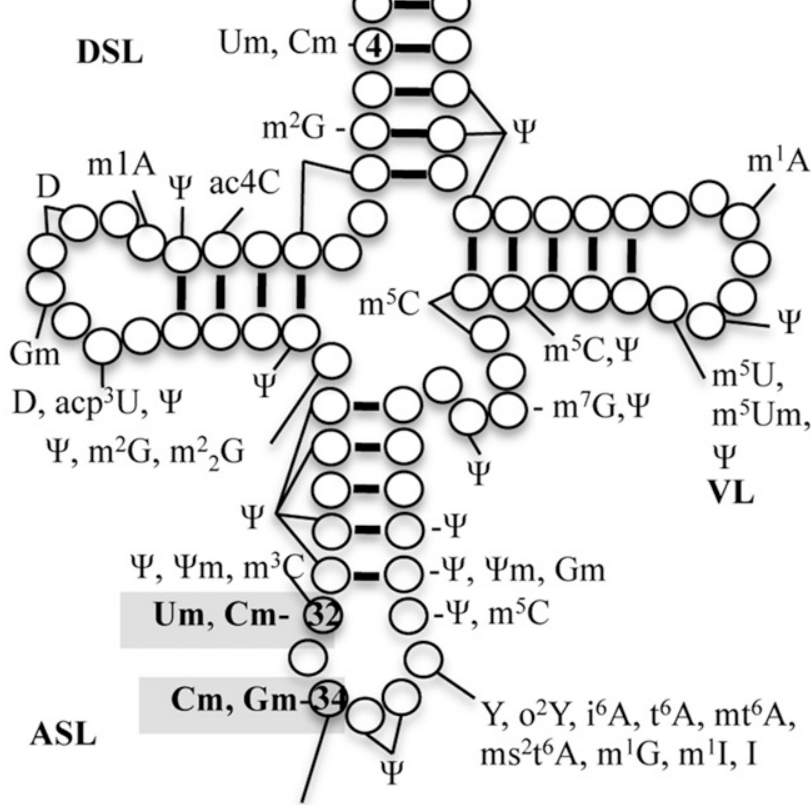

$\mathrm{m}^{5} \mathrm{C}, \mathrm{mcm}^{5} \mathrm{U}, \mathrm{mcm}^{5} \mathrm{~s}^{2} \mathrm{U}, \mathrm{s}^{2} \mathrm{U}, \mathrm{Q}, \mathrm{manQ}, \mathrm{I}$

B

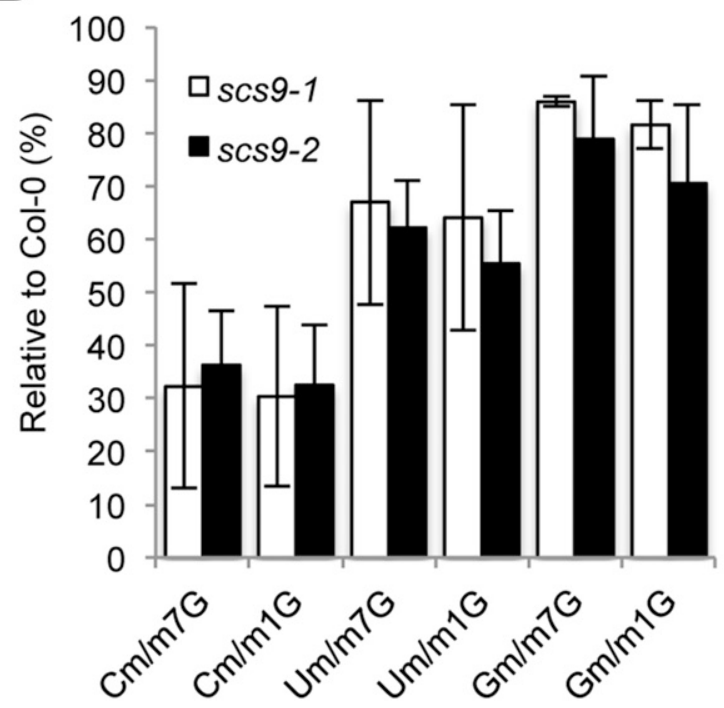

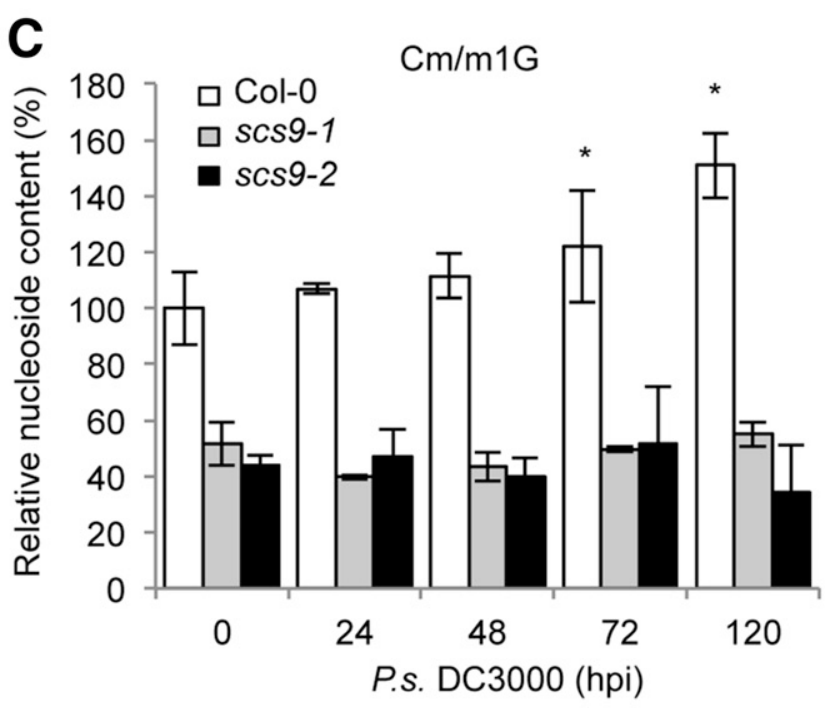

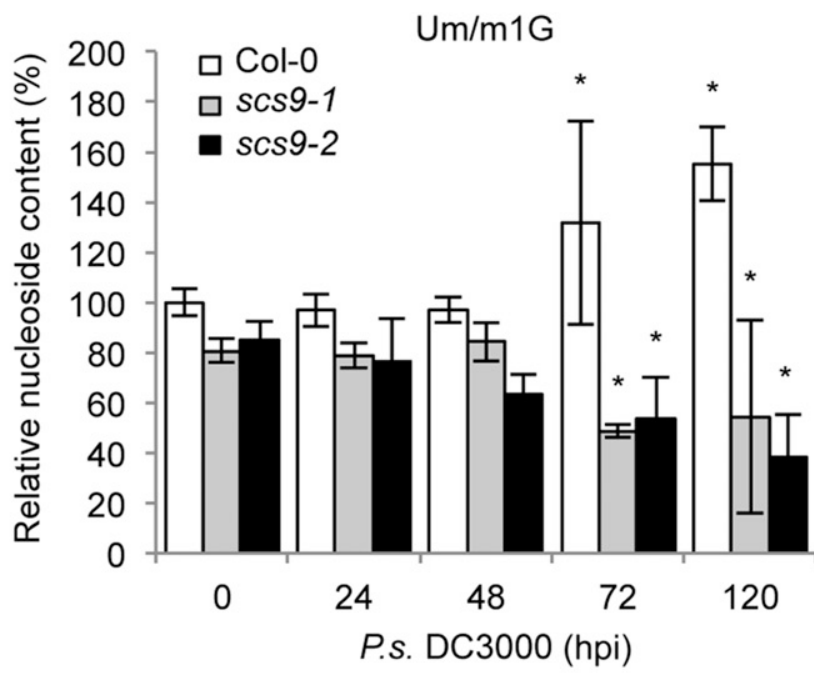

D

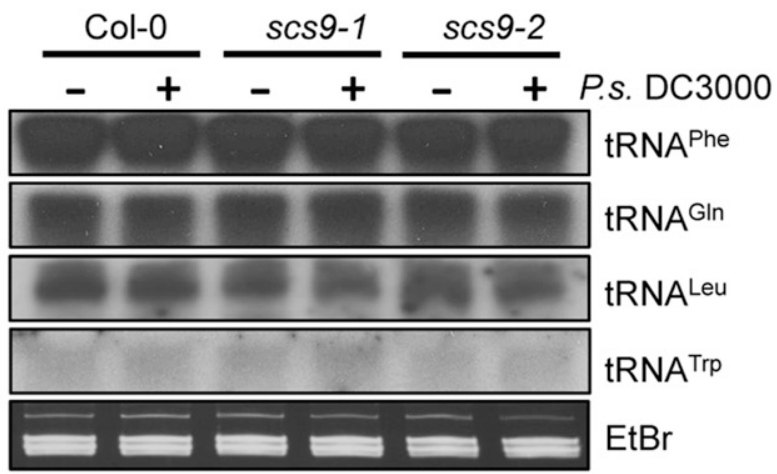


Our results also led us to hypothesize that the absence of specific methylation at the wobble position of the selective group of tRNAs controlled by SCS9 might affect the synthesis of one or more codon-biased cognate proteins required for resistance to DC3000, which might function as repressor proteins. If the repressor is defective or poorly translated, then a cellular factor or molecular process might become derepressed, which in turn might favor bacterial growth and host colonization. This may reconcile with the existence of tRNA cleaving toxins, developed by certain microbes to act on specific positions within the anticodon loop of target tRNAs, such as colicin and onconase (Phizicky and Hopper 2010) or the $\gamma$-toxin of Kluyveromyces lactis that targets and cleaves specific tRNAs in the yeast $S$. cerevisiae that have the $\mathrm{mcm}^{5} \mathrm{~s}^{2} \mathrm{U}$ modification at position 34 of tRNA ${ }^{\text {Glu(UUU) }}$ (Lu et al. 2005). At this stage, we cannot disregard the idea that bacterial effector proteins may have been developed to target specific modified plant tRNAs or even the MTase responsible for such tRNA modification as part of a pathogenic strategy.

We cannot ignore the possibility of a surveillance mechanism leading to hypomodified tRNA degradation as part of a conserved response to stress (Kadaba et al. 2004; Motorin and Helm 2010), which might have a potential impact on translation and disease progression, as described by Thompson and Parker (2009). The emergence of tRNA-derived fragments as a novel class of small regulatory RNAs (Lee et al. 2009; Pederson 2010) with potential roles as regulators of gene silencing and genome stability, through the targeting of transposable element transcripts (Maute et al. 2013; Raina and Ibba 2014; Schorn et al. 2017), and, as part of a conserved stress response also described in plants (Martinez et al. 2017; Thompson et al. 2008), might therefore interfere with epigenetic processes governed by small RNAs, such as RNA-directed DNA methylation, which has recently been demonstrated as pivotal for plant immunity to DC3000 (López et al. 2011). However, this latter mechanism seems less likely, since the hypomodified tRNA levels in $\operatorname{scs} 9$ plants appeared not to be degraded, not even following inoculation with the pathogen.

In summary, the present results denote the importance of keeping intact tRNA modifications for mounting an effective disease resistance response toward DC300. Unraveling further components, its regulation, and identifying target proteins whose translation is regulated by these tRNAs modifications will open new avenues to better understand how the effectiveness of disease resistance is regulated in plants.

\section{MATERIALS AND METHODS}

\section{Plant growth conditions.}

Arabidopsis thaliana plants were grown in a growth chamber (19 to $23^{\circ} \mathrm{C}$ ) on a 10 -h light and 14 -h dark cycle as previously described (Ramírez et al. 2013). All mutants are in Col-0 background.

\section{$\beta$-Glucuronidase (GUS) staining and callose deposition analysis.}

Protocols for GUS staining and determination of callose deposition in plant leaves were performed as previously described (Coego et al. 2005; García-Andrade et al. 2013).

\section{Mapping and cloning.}

The $\operatorname{scs} 9$ mutant was backcrossed twice to the $\operatorname{csb} 3$ line to confirm its recessive inheritance; $\operatorname{scs} 9$ plants were crossed to Ler; and F1 plants were allowed to self. F2 plants were scored for cosegregation of enhanced disease susceptibility to DC3000 with SSLPs (Bell and Ecker 1994; López et al. 2011). Molecular markers were derived from the polymorphism database between the Ler and Col-0 ecotypes (The Arabidopsis Information Resource website). Whole-genome sequencing and identification of nucleotide polymorphisms was carried out at the John Innes Centre (Norwich, U.K.) as a genome service exchange, using an Illumina GAIIx platform and the bioinformatic pipeline devised by Austin et al. (2011).

\section{Gene constructs and expression in yeast and transgenic plants.}

To amplify SCS9 cDNA, PCR was performed using the Expand high-fidelity PCR system (Roche), with $1 \mu \mathrm{l}$ of cDNA, using BPSCS9F $w$ and BPSCS9Rv specific primers including Gateway adapters and recombined to $p D O N R 221$ using Gateway technology (Life Technologies) and were recombined into the different destination vectors using LR ClonaseMixII kit (Invitrogen). For the SCS9-GFP overexpressing construct, pDONR221 SCS9 was recombined to $p B 7 F W G$ destination. For the SCS9-mCherry overexpressing construct, $p D O N R 221$ SCS9 was recombined to the modified pEarlyGate101 vector (García-Andrade et al. 2013). For the yeast trm7 complementation assay, pDONR221 SCS9 vector was recombined to the yeast expression vectors $p 423-G A L 1$ and $p 423-G A L 1-G F P$, to generate $p 423$ GAL1/SCS9 and p423 GAL1/SCS9-GFP, respectively. Individual clones were sequenced to confirm absence of errors in the SCS9 cDNA. p423 GAL1/SCS9, $p 423$ GAL1/SCS9-GFP, and the respective empty vectors were transformed into wild type (BMA64, MAT $\alpha$ [Pintard et al.

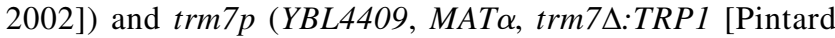
et al. 2002]) yeast strains as previously described (Castelló et al. 2011) and were grown on agar plates lacking His. Transformants were then transferred to selective liquid media containing 2\% (wt/vol) Gal to induce expression of SCS9. These cultures were incubated at $28^{\circ} \mathrm{C}$ for 2 days, and then, were adjusted to an optical density at $600 \mathrm{~nm}\left(\mathrm{OD}_{600}\right)=0.1$ to perform the time-course growing assays.

Fig. 6. Reduced content of transfer RNA (tRNA)-derived $2^{\prime}$ - $O$-methylcytosine $(\mathrm{Cm}), 2^{\prime}-O$-methyluracil (Um), and $2^{\prime}$ - $O$-methylguanine $(\mathrm{Gm})$ in $s c s 9-1$ and scs 9 -2 mutants. A, Position of common modifications found in cytoplasmic tRNA in Arabidopsis and its major domains. tRNA is shown in its common secondary structure form, with circles representing nucleotides and lines representing base pairs. Positions in shaded gray indicate the proposed tRNA methylation site for Trm7-like MTases in eukaryotes. Residue 34 corresponds to the wobble nucleotide. AAS = amino acid-accepting stem, DSL = dihydrouridine stem and loop, ASL = anticodon stem loop, and VL = variable loop. Conventional abbreviations for nucleotide modifications are used (the Modomics database). B, Percent reduction of $\mathrm{Cm}$, Um, and $\mathrm{Gm}$ content in scs $9-1$ and scs $9-2$ relative to Col-0 plants as determined by high-pressure liquid chromatography analysis. Each value was relative to the amount of tRNA-derived 7 methylguanosine ( $\left.\mathrm{m}^{7} \mathrm{G}\right)$ and $1-\mathrm{methylguanosine}$ ( $\left.\mathrm{m}^{1} \mathrm{G}\right)$, which remained invariant in all the genotypes. Data points represent the average of three biological replicates. C, Relative content of Cm- and Um-modified nucleosides in Col-0, scs9-1, and scs9-2 plants during the course of an infection with Pseudomonas syringae DC3000, as determined by liquid chromatography-mass spectrometry. Samples were taken at $0,24,48,72$, and 120 h postinoculation (hpi). Each value was relative to the amount of tRNA-derived $\mathrm{m}^{1} \mathrm{G}$, which remained invariant during the infection process. Data represent the mean \pm standard deviation; $n=3$. Asterisks indicate statistical differences compared with its respective genotype as referred to its time 0 control $(P<0.05)$, analyzed using a Student's $t$ test. D, tRNA Northern blot in mock- and DC3000-inoculated Col-0, scs9-1, and $s c s 9$-2 plants. Leaf samples were collected at 3 days postinoculation. The membranes were hybridized with radiolabel probes to tRNAPhe, tRNAGln, tRNALeu, tRNATrp. Equal RNA loading was verified by staining with $\mathrm{EtBr}$ (bottom panel). 
Transient expression in Nicotiana benthamiana leaves and confocal laser-scanning microscopy.

Fully expanded leaves of $N$. benthamiana were agroinfiltrated with a suspension of Agrobacterium tumefaciens C58 bearing the relevant construct, and this plant tissue was analyzed with a Leica TCS LS spectral confocal microscope as previously described (Dobón et al. 2015).

\section{RT-qPCR.}

qPCR assays were performed, using an ABI PRISM 7000 sequence detection system and SYBR Green (Perkin-Elmer Applied Biosystems), as described previously (García-Andrade et al. 2013). ACTIN2 was used as a reference gene.

\section{Western blots.}

Protein crude extracts, SDS-polyacrylamide gel electrophoresis, and immunoblots were performed as previously described (Dobón et al. 2015).

\section{Pseudomonas syringae inoculations.}

Arabidopsis leaves were inoculated with DC3000 as previously described (Coego et al. 2005; Gil et al. 2005; López et al. 2011; Ramírez et al. 2010, 2013), using a bacterial inoculum at a final $\mathrm{OD}_{600}$ of 0.1 , with $0.02 \%$ Silwet L-77 (Crompton Europe) if inoculated by spray or with a further 1:500 dilution (without Silwett L-77) when inoculation was performed by leaf infiltration with a syringe.

\section{H. arabidopsidis assays.}

$H$. arabidopsidis WACO9 was cultivated in susceptible $N a h G$ plants in Wassileswskija background. The spores were collected by washing the sporulating tissue with autoclaved water and were subsequently cleaned by filtering using two Miracloth layers. The inoculum was prepared by measuring and adjusting the spore concentration using a Neubauer chamber. For the mutant analysis, 3-week-old Arabidopsis seedlings were inoculated with a suspension of water containing $H$. arabidopsidis spores at a concentration of $10^{5}$ spores per milliliter and were kept in high humidity conditions. Six days after spraying, around 200 infected leaves per genotype, from a population of around 50 seedlings, were lactophenol trypan blue-stained (100 mg of trypan blue, $50 \mathrm{mg}$ of phenol, $50 \mathrm{mg}$ of glycerol $100 \%, 50 \mathrm{ml}$ of lactic acid, water to $150 \mathrm{ml}$ volume, plus $300 \mathrm{ml}$ of Ethanol Absolute), and were preserved in $60 \%$ chloral hydrate. The disease progression was scored by direct visualization of the samples using a stereomicroscope. Infected leaves were assigned to four different categories based on the degree of infection: class I = healthy leaves, class II = discrete pathogen growth and no sporulation, class III = high degree of pathogen growth and reduce sporulation (from 1 to 5 spores), and class IV = high degree of pathogen growth and sporulation.

\section{T-DNA Arabidopsis mutants.}

T-DNA insertion mutants and primers used to identify insertions by PCR are listed in Supplementary Table S3.

tRNA isolation, digestion, HPLC, and LC-MS analysis.

Total RNA was extracted using Trizol reagent (Invitrogen), small RNAs were separated from ribosomal RNA and mRNA using $\mathrm{LiCl}$, and tRNA was further purified using cellulose DE52 resin (Whatman) as described by Chen et al. (2010). tRNA $(100 \mu \mathrm{g})$ dissolved in Milli-Q water was degraded to nucleosides with P1 nuclease (Sigma Aldrich) and alkaline phosphatase (Toyobo), as described by Chen et al. (2010). The modified nucleosides were analyzed using Reverse-phase HPLC (Waters Alliance HPLC system and Waters Absorbance Detector 2996) and a C-30 column (Develosil C-30 reverse-phase column, $250 \times 4.6 \mathrm{~mm}$; Nomura Chemical Co., Ltd.). The buffer gradient was as described (Chen et al. 2010). Modified nucleosides were quantified relative to two internal standards $\left(\mathrm{m}^{7} \mathrm{G}\right.$ and $\left.\mathrm{m}^{1} \mathrm{G}\right)$. For LC-MS analysis, total RNA and microRNA was extracted using a microRNA extraction kit (Omega Bio-Tek Inc.). RNA concentration was determined using a NanoDrop ND-1000 spectrophotometer (Thermo Scientific), about $20 \mu \mathrm{g}$ tRNA was digested with 2 U P1 nuclease (Sigma) and $1.5 \mathrm{U}$ of calf-intestine alkaline phosphatase (Toyobo) in $20 \mu \mathrm{l}$ of $20 \mathrm{mM}$ HEPES-KOH (pH 7.0) at $37^{\circ} \mathrm{C}$ for $3 \mathrm{~h}$. Sample was diluted with MilliQ water (Millipore Synnergy) to a concentration of $5 \mathrm{ug} / \mathrm{ml}$, the injected volume was $10 \mu \mathrm{l}$. API 4000 Q-TRAP mass spectrometer (Applied Biosystems) was used with an LC-20A HPLC system and a diode array UV detector (190 to $400 \mathrm{~nm}$ ) equipped with an electro spray ionization source. Electrospray ionization MS was conducted in a positive ion mode. The nebulizer gas, auxiliary gas, curtain gas, turbo gas temperature, entrance potential, and ion spray voltage were $60 \mathrm{psi}, 65 \mathrm{psi}, 15 \mathrm{psi}, 550^{\circ} \mathrm{C}, 10 \mathrm{~V}$, and 5,500 $\mathrm{V}$, respectively. Multiple reaction monitoring mode was performed to determine parent-to-product ion transitions. An Inertsil ODS-3 $(2.1 \mathrm{~mm} \times 150 \mathrm{~mm}, 5 \mu \mathrm{m}$ particle size; Shimadzu) reversed-phase column with an Inertsil ODS guard column $(4 \mathrm{~mm} \times 10 \mathrm{~mm}, 5 \mu \mathrm{m}$ particle size; Shimadzu) was used for chromatographic separation of nucleosides. The mobile phase gradient consisted of $2 \mathrm{mM}$ ammonium acetate (solvent A) and methanol (solvent B). The flow rate was $0.6 \mathrm{ml} / \mathrm{min}$ at ambient temperature. Nucleoside standard uridine, cytidine, adenosine, guanosine, 1-methyladenosine, 7methylguanosine, 5-methyluridine and $2^{\prime}$ - $O$-methylguanosine nucleoside standards (Santa Cruz Biotechnology) were used to distinguish nucleoside isomers. The relative abundance of each selected modified nucleoside was calculated as area of the peak with the correct mass and parent-to-product ion transition divided by total area for uridine, cytidine, adenosine, and guanosine monitored nucleosides. Then, the relative content of $\mathrm{Cm}, \mathrm{Um}$, and $\mathrm{Gm}$ nucleosides was related to one or both $\mathrm{m}^{1} \mathrm{G}$ and $\mathrm{m}^{7} \mathrm{G}$.

\section{Anthocyanin and SA quantification.}

Anthocyanin extraction and quantification was performed as previously described (Ramírez et al. 2010). SA extraction and quantification was performed as described (Defraia et al. 2008; Huang et al. 2005).

\section{Paromomycin and rose bengal assays.}

Seeds were sterilized and stratified in darkness at $4{ }^{\circ} \mathrm{C}$ for 3 days and were transferred to growth chambers for each assay. To conduct paromomycin rate assay, seeds were plated on Murashige and Skoog medium (Duchefa Biochemie) supplemented with $0.5 \%$ sucrose, without or with paromomycin. Pictures were taken after 14 days. To conduct the rose bengal assay, seeds sown on Murashige and Skoog plates were vertically grown for 4 days under normal conditions, and then, seedlings were transferred to vertical square MS plates with or without $2 \mu \mathrm{M}$ rose bengal. The pictures were taken after 7 days.

\section{tRNA blot analysis.}

Total RNA was extracted from leaf tissue using TRIzol (Invitrogen), following the manufacturer's protocol. A total of $4 \mu \mathrm{g}$ of RNA was electrophoresed $15 \%$ (wt/vol) polyacrylamide gels under denaturing conditions and was then transferred to Hybond N (Amersham) membranes. Oligonucleotide probes were 32P-labeled with T4 polynucleotide kinase (Fermentas), according to manufacturer's instructions, and were then purified on a microspin G-25 column, separating labeled probe from unincorporated nucleotides. The specific activity was 
determined using scintillation counter. Prehybridization of the membranes was carried out for $30 \mathrm{~min}$ in Church hybridization buffer (0.5 M NaPO4 [pH 7.2], 7\% [wt/vol] SDS). Hybridization was carried out in the same buffer overnight at $40^{\circ} \mathrm{C}$, followed by three washes at the same temperature for $20 \mathrm{~min}$ each in $0.5 \times \mathrm{SSC} / 0.1 \% \mathrm{SDS}(1 \times \mathrm{SSC}$ is $0.15 \mathrm{M} \mathrm{NaCl}$ plus 0.015 M sodium citrate) (Church and Gilbert 1984).

\section{Flg22 treatments and $\mathrm{NaCl}$ growth assay.}

For flg22 treatment, seedlings were grown and treated with $1 \mu \mathrm{M}$ flg 22 as previously described (García-Andrade et al. 2011). For saline growth assay and effect on germination, sterilized seeds were plated on Murashige and Skoog solid supplemented with $0.5 \%$ sucrose and morpholineethanesulfonic acid (MES) $(0.01 \%)$, without or with $\mathrm{NaCl}$ (at different concentrations). Pictures and fresh weight data were taken after 13 days. To analyze seedling growth inhibition by salt stress, seeds sown and grown under normal conditions (without $\mathrm{NaCl}$ ) for 6 days were transferred to Murashige and Skoog medium without and with $\mathrm{NaCl}$ (at different concentrations). Pictures and data of fresh weight were taken after 7 days of treatment; 20 seedlings were analyzed for each replicate. For seedling growth-inhibition assays by flg22, 7-day-old seedlings, grown in Murashige and Skoog solid plates (with $0.5 \%$ sucrose and $0.01 \%$ MES), were transferred to Murashige and Skoog liquid media, with or without $1 \mu \mathrm{M}$ flg22, and were softly shaken $(90 \mathrm{rpm})$ for 5 days, and the fresh weight from groups of 10 seedling of each genotype was measured.

\section{ACKNOWLEDGMENTS}

We acknowledge B. Lapeyre for providing $S$. cereviseae trm $7 \Delta$ mutant strain and plasmids, G. Etherington and B. Wulff for assistance in genome sequencing of $\operatorname{scs} 9$ mutant, P. Tornero for the Pseudomonas syringae, $\mathrm{HrpC}$, and Acinetobacter strains and assistance in SA determination, L. Pérez for her assistance on the identification of the initial $\operatorname{csb} 3 \operatorname{scs} 9$ mutant.

\section{LITERATURE CITED}

Agris, P. F., Vendeix, F. A., and Graham, W. D. 2007. tRNA's wobble decoding of the genome: 40 years of modification. J. Mol. Biol. 366:1-13.

Asai, T., Tena, G., Plotnikova, J., Willmann, M. R., Chiu, W. L., GomezGomez, L., Boller, T., Ausubel, F. M., and Sheen, J. 2002. MAP kinase signalling cascade in Arabidopsis innate immunity. Nature 415:977-983.

Austin, R. S., Vidaurre, D., Stamatiou, G., Breit, R., Provart, N. J., Bonetta D., Zhang, J., Fung, P., Gong, Y., Wang, P. W., McCourt, P., and Guttman, D. S. 2011. Next-generation mapping of Arabidopsis genes. Plant J. 67:715-725.

Begley, U., Dyavaiah, M., Patil, A., Rooney, J. P., DiRenzo, D., Young, C. M., Conklin, D. S., Zitomer, R. S., and Begley, T. J. 2007. Trm9catalyzed tRNA modifications link translation to the DNA damage response. Mol. Cell 28:860-870.

Bell, C. J., and Ecker, J. R. 1994. Assignment of 30 microsatellite loci to the linkage map of Arabidopsis. Genomics 19:137-144.

Bethke, G., Unthan, T., Uhrig, J. F., Pöschl, Y., Gust, A. A., Scheel, D., and Lee, J. 2009. Flg22 regulates the release of an ethylene response factor substrate from MAP kinase 6 in Arabidopsis thaliana via ethylene signaling. Proc. Natl. Acad. Sci. U.S.A. 106:8067-8072.

Björk, G. R. 1995. Biosynthesis and function of modified nucleosides. Pages 165-205 in: tRNA: Structure, Biosynthesis and Function. D. Soll and U. L. RajBhandary, ed. American Society for Microbiology, Washington, D.C.

Bügl, H., Fauman, E. B., Staker, B. L., Zheng, F., Kushner, S. R., Saper, M. A., Bardwell, J. C., and Jakob, U. 2000. RNA methylation under heat shock control. Mol. Cell 6:349-360.

Bujnicki, J. M. and Rychlewski, L. 2001. Reassignment of specificities of two cap methyltransferase domains in the reovirus lambda 2 protein. Genome Biol. 2:research0038.1.

Burgess, A., David, R., and Searle, I. R. 2016. Deciphering the epitranscriptome: A green perspective. J. Integr. Plant Biol. 58:822-835.

Burgess, A. L., David, R., and Searle, I. R. 2015. Conservation of tRNA and rRNA 5-methylcytosine in the kingdom Plantae. BMC Plant Biol. 15:199.

Caldas, T., Binet, E., Bouloc, P., Costa, A., Desgres, J., and Richarme, G. 2000. The FtsJ/RrmJ heat shock protein of Escherichia coli is a $23 \mathrm{~S}$ ribosomal RNA methyltransferase. J. Biol. Chem. 275:16414-16419.
Cao, H., Glazebrook, J., Clarke, J. D., Volko, S., and Dong, X. 1997. The Arabidopsis NPR1 gene that controls systemic acquired resistance encodes a novel protein containing ankyrin repeats. Cell 88:57-63.

Castelló, M. J., Carrasco, J. L., Navarrete-Gómez, M., Daniel, J., Granot, D., and Vera, P. 2011. A plant small polypeptide is a novel component of DNA-binding protein phosphatase 1-mediated resistance to Plum pox virus in Arabidopsis. Plant Physiol. 157:2206-2215.

Chan, C. T., Dyavaiah, M., DeMott, M. S., Taghizadeh, K., Dedon, P. C., and Begley, T. J. 2010. A quantitative systems approach reveals dynamic control of tRNA modifications during cellular stress. PLoS Genet. 6: e1001247.

Chan, C. T., Pang, Y. L., Deng, W., Babu, I. R., Dyavaiah, M., Begley, T. J., and Dedon, P. C. 2012. Reprogramming of tRNA modifications controls the oxidative stress response by codon-biased translation of proteins Nat. Commun. 3:937.

Chen, P., Jäger, G., and Zheng, B. 2010. Transfer RNA modifications and genes for modifying enzymes in Arabidopsis thaliana. BMC Plant Biol. 10:201

Chernoff, Y. O., Vincent, A., and Liebman, S. W. 1994. Mutations in eukaryotic $18 \mathrm{~S}$ ribosomal RNA affect translational fidelity and resistance to aminoglycoside antibiotics. EMBO J. 13:906-913.

Church, G. M., and Gilbert, W. 1984. Genomic sequencing. Proc. Natl. Acad. Sci. U.S.A. 81:1991-1995

Clay, N. K., Adio, A. M., Denoux, C., Jander, G., and Ausubel, F. M. 2009. Glucosinolate metabolites required for an Arabidopsis innate immune response. Science 323:95-101.

Coego, A., Ramirez, V., Ellul, P., Mayda, E., and Vera, P. 2005. The $\mathrm{H}_{2} \mathrm{O}_{2^{-}}$ regulated $E p 5 C$ gene encodes a peroxidase required for bacterial speck susceptibility in tomato. Plant J. 42:283-293.

David, R., Burgess, A., Parker, B., Li, J., Pulsford, K., Sibbritt, T., Preiss, T., and Searle, I. R. 2017. Transcriptome-wide mapping of RNA 5methylcytosine in Arabidopsis mRNAs and noncoding RNAs. Plant Cell 29:445-460.

Dedon, P. C., and Begley, T. J. 2014. A system of RNA modifications and biased codon use controls cellular stress response at the level of translation. Chem. Res. Toxicol. 27:330-337.

Defraia, C. T., Schmelz, E. A., and Mou, Z. 2008. A rapid biosensor-based method for quantification of free and glucose-conjugated salicylic acid. Plant Methods 4:28.

Deng, W.-L., Preston, G., Collmer, A., Chang, C.-J., and Huang, H.-C. 1998. Characterization of the hrpC and hrpRS operons of Pseudomonas syringae pathovars syringae, tomato, and glycinea and analysis of the ability of $h r p F, h r p G, h r c C$, $h r p T$, and $h r p V$ mutants to elicit the hypersensitive response and disease in plants. J. Bacteriol. 180 4523-4531.

Dobón, A., Canet, J. V., García-Andrade, J., Angulo, C., Neumetzler, L., Persson, S., and Vera, P. 2015. Novel disease susceptibility factors for fungal necrotrophic pathogens in Arabidopsis. PLoS Pathog. 11: e1004800.

El Yacoubi, B., Bailly, M., and de Crécy-Lagard, V. 2012. Biosynthesis and function of posttranscriptional modifications of transfer RNAs. Annu. Rev. Genet. 46:69-95.

García-Andrade, J., Ramírez, V., Flors, V., and Vera, P. 2011. Arabidopsis ocp3 mutant reveals a mechanism linking ABA and JA to pathogeninduced callose deposition. Plant J. 67:783-794.

García-Andrade, J., Ramírez, V., López, A., and Vera, P. 2013. Mediated plastid RNA editing in plant immunity. PLoS Pathog. 9:e1003713.

Gil, M. J., Coego, A., Mauch-Mani, B., Jordá, L., and Vera, P. 2005. The Arabidopsis $c s b 3$ mutant reveals a regulatory link between salicylic acidmediated disease resistance and the methyl-erythritol 4-phosphate pathway. Plant J. 44:155-166.

Gu, C., Begley, T. J., and Dedon, P. C. 2014. tRNA modifications regulate translation during cellular stress. FEBS Lett. 588:4287-4296.

Guy, M. P., Podyma, B. M., Preston, M. A., Shaheen, H. H., Krivos, K. L., Limbach, P. A., Hopper, A. K., and Phizicky, E. M. 2012. Yeast Trm7 interacts with distinct proteins for critical modifications of the tRNAPhe anticodon loop. RNA 18:1921-1933.

Hori, H. 2014. Methylated nucleosides in tRNA and tRNA methyltransferases. Front. Genet. 5:144.

Huang, H.-Y., and Hopper, A. K. 2016. Multiple layers of stress-induced regulation in tRNA biology. Life (Basel) 6:16.

Huang, W. E., Wang, H., Zheng, H., Huang, L., Singer, A. C., Thompson, I., and Whiteley, A. S. 2005. Chromosomally located gene fusions constructed in Acinetobacter sp. ADP1 for the detection of salicylate. Environ. Microbiol. 7:1339-1348.

Jackman, J. E., Montange, R. K., Malik, H. S., and Phizicky, E. M. 2003. Identification of the yeast gene encoding the tRNA $\mathrm{m}^{1} \mathrm{G}$ methyltransferase responsible for modification at position 9. RNA 9:574-585. 
Jordá, L., and Vera, P. 2000. Local and systemic induction of two defenserelated subtilisin-like protease promoters in transgenic Arabidopsis plants. Luciferin induction of PR gene expression. Plant Physiol. 124: 1049-1058.

Jühling, F., Mörl, M., Hartmann, R. K., Sprinzl, M., Stadler, P. F., and Pütz, J. 2009. tRNAdb 2009: compilation of tRNA sequences and tRNA genes. Nucleic Acids Res. 37:D159-D162.

Kadaba, S., Krueger, A., Trice, T., Krecic, A. M., Hinnebusch, A. G., and Anderson, J. 2004. Nuclear surveillance and degradation of hypomodified initiator tRNAMet in S. cerevisiae. Genes Dev. 18:1227-1240.

Kalhor, H. R., and Clarke, S. 2003. Novel methyltransferase for modified uridine residues at the wobble position of tRNA. Mol. Cell. Biol. 23: 9283-9292.

Khoury, C. M., Yang, Z., Li, X. Y., Vignali, M., Fields, S., and Greenwood, M. T. 2008. A TSC22-like motif defines a novel antiapoptotic protein family. FEMS Yeast Res. 8:540-563.

Kirchner, S., and Ignatova, Z. 2015. Emerging roles of tRNA in adaptive translation, signalling dynamics and disease. Nat. Rev. Genet. 16: 98-112.

Kramer, E. B., and Hopper, A. K. 2013. Retrograde transfer RNA nuclear import provides a new level of tRNA quality control in Saccharomyces cerevisiae. Proc. Natl. Acad. Sci. U.S.A. 110:21042-21047.

Kuchino, Y., Borek, E., Grunberger, D., Mushinski, J. F., and Nishimura, S. 1982. Changes of post-transcriptional modification of wye base in tumor-specific tRNAPhe. Nucleic Acids Res. 10:6421-6432.

Lee, Y. S., Shibata, Y., Malhotra, A., and Dutta, A. 2009. A novel class of small RNAs: tRNA-derived RNA fragments (tRFs). Genes Dev. 23: 2639-2649.

López, A., Ramírez, V., García-Andrade, J., Flors, V., and Vera, P. 2011. The RNA silencing enzyme RNA polymerase $\mathrm{v}$ is required for plant immunity. PLoS Genet. 7:e1002434.

Lu, J., Huang, B., Esberg, A., Johansson, M. J., and Byström, A. S. 2005. The Kluyveromyces lactis $\gamma$-toxin targets tRNA anticodons. RNA 11: 1648-1654.

Machnicka, M. A., Milanowska, K., Osman Oglou, O., Purta, E., Kurkowska, M., Olchowik, A., Januszewski, W., Kalinowski, S., Dunin-Horkawicz, S., Rother, K. M., Helm, M., Bujnicki, J. M., and Grosjean, H. 2013. MODOMICS: a database of RNA modification pathways-2013 update. Nucleic Acids Res. 41:D262-D267.

Machnicka, M. A., Olchowik, A., Grosjean, H., and Bujnicki, J. M. 2014. Distribution and frequencies of post-transcriptional modifications in tRNAs. RNA Biol. 11:1619-1629.

Martinez, G., Choudury, S. G., and Slotkin, R. K. 2017. tRNA-derived small RNAs target transposable element transcripts. Nucleic Acids Res. 45: 5142-5152.

Mauro, V. P., and Edelman, G. M. 2007. The ribosome filter redux. Cell Cycle 6:2246-2251.

Maute, R. L., Schneider, C., Sumazin, P., Holmes, A., Califano, A., Basso, K., and Dalla-Favera, R. 2013. tRNA-derived microRNA modulates proliferation and the DNA damage response and is down-regulated in B cell lymphoma. Proc. Natl. Acad. Sci. U.S.A. 110:1404-1409.

Miyawaki, K., Tarkowski, P., Matsumoto-Kitano, M., Kato, T., Sato, S., Tarkowska, D., Tabata, S., Sandberg, G., and Kakimoto, T. 2006. Roles of Arabidopsis ATP/ADP isopentenyltransferases and tRNA isopentenyltransferases in cytokinin biosynthesis. Proc. Natl. Acad. Sci. U.S.A. 103:16598-16603.

Motorin, Y., and Helm, M. 2010. tRNA stabilization by modified nucleotides. Biochemistry 49:4934-4944.

Nawrath, C., and Métraux, J. P. 1999. Salicylic acid induction-deficient mutants of Arabidopsis express PR-2 and PR-5 and accumulate high levels of camalexin after pathogen inoculation. Plant Cell 11:1393-1404.

Novoa, E. M., Pavon-Eternod, M., Pan, T., and Ribas de Pouplana, L. 2012. A role for tRNA modifications in genome structure and codon usage. Cell 149:202-213.

Pajerowska-Mukhtar, K. M., Wang, W., Tada, Y., Oka, N., Tucker, C. L., Fonseca, J. P., and Dong, X. 2012. The HSF-like transcription factor TBF1 is a major molecular switch for plant growth-to-defense transition. Curr. Biol. 22:103-112.
Pederson, T. 2010. Regulatory RNAs derived from transfer RNA? RNA 16: 1865-1869.

Phizicky, E. M., and Hopper, A. K. 2010. tRNA biology charges to the front. Genes Dev. 24:1832-1860.

Pintard, L., Lecointe, F., Bujnicki, J. M., Bonnerot, C., Grosjean, H., and Lapeyre, B. 2002. Trm7p catalyses the formation of two 2'-Omethylriboses in yeast tRNA anticodon loop. EMBO J. 21:1811-1820.

Pósfai, J., Bhagwat, A. S., Pósfai, G., and Roberts, R. J. 1989. Predictive motifs derived from cytosine methyltransferases. Nucleic Acids Res. 17: 2421-2435.

Raina, M., and Ibba, M. 2014. tRNAs as regulators of biological processes. Front. Genet. 5:171.

Ramírez, V., López, A., Mauch-Mani, B., Gil, M. J., and Vera, P. 2013. An extracellular subtilase switch for immune priming in Arabidopsis. PLoS Pathog. 9:e1003445.

Ramírez, V., Van der Ent, S., García-Andrade, J., Coego, A., Pieterse, C. M., and Vera, P. 2010. OCP3 is an important modulator of NPR1mediated jasmonic acid-dependent induced defenses in Arabidopsis. BMC Plant Biol. 10:199.

Różanowska, M., Ciszewska, J., Korytowski, W., and Sarna, T. 1995. Rosebengal-photosensitized formation of hydrogen peroxide and hydroxyl radicals. J. Photochem. Photobiol. B 29:71-77.

Schorn, A. J., Gutbrod, M. J., LeBlanc, C., and Martienssen, R. 2017. LTRretrotransposon control by tRNA-derived small RNAs. Cell 170:P61-71. E11.

Takano, K., Nakagawa, E., Inoue, K., Kamada, F., Kure, S., and Goto, Y.; Japanese Mental Retardation Consortium. 2008. A loss-of-function mutation in the FTSJ1 gene causes nonsyndromic X-linked mental retardation in a Japanese family. Am. J. Med. Genet. B. Neuropsychiatr. Genet. 147B:479-484.

Thompson, D. M., Lu, C., Green, P. J., and Parker, R. 2008. tRNA cleavage is a conserved response to oxidative stress in eukaryotes. RNA 14: 2095-2103.

Thompson, D. M., and Parker, R. 2009. Stressing out over tRNA cleavage. Cell 138:215-219.

Walden, T. L., Jr., Howes, N., and Farkas, W. R. 1982. Purification and properties of guanine, queuine-tRNA transglycosylase from wheat germ. J. Biol. Chem. 257:13218-13222.

Wang, X., and He, C. 2014. Dynamic RNA modifications in posttranscriptional regulation. Mol. Cell 56:5-12.

Wang, Y., Li, D., Gao, J., Li, X., Zhang, R., Jin, X., Hu, Z., Zheng, B., Persson, S., and Chen, P. 2017. The $2^{\prime}$ - $O$-methyladenosine nucleoside modification gene OsTRM13 positively regulates salt stress tolerance in rice. J. Exp. Bot. 68:1479-1491.

Wilkinson, M. L., Crary, S. M., Jackman, J. E., Grayhack, E. J., and Phizicky, E. M. 2007. The 2'-O-methyltransferase responsible for modification of yeast tRNA at position 4. RNA 13:404-413.

Xu, G., Greene, G. H., Yoo, H., Liu, L., Marqués, J., Motley, J., and Dong, X. 2017a. Global translational reprogramming is a fundamental layer of immune regulation in plants. Nature 545:487-490.

Xu, G., Yuan, M., Ai, C., Liu, L., Zhuang, E., Karapetyan, S., Wang, S., and Dong, X. 2017b. uORF-mediated translation allows engineered plant disease resistance without fitness costs. Nature 545:491-494.

Yan, S., and Dong, X. 2014. Perception of the plant immune signal salicylic acid. Curr. Opin. Plant Biol. 20:64-68.

Yewdell, J. W., Antón, L. C., and Bennink, J. R. 1996. Defective ribosomal products (DRiPs): A major source of antigenic peptides for MHC class I molecules? J. Immunol. 157:1823-1826.

Zhou, W., Karcher, D., and Bock, R. 2013. Importance of adenosine-toinosine editing adjacent to the anticodon in an Arabidopsis alanine tRNA under environmental stress. Nucleic Acids Res. 41:3362-3372.

\section{AUTHOR-RECOMMENDED INTERNET RESOURCES}

The Arabidopsis Information Resource website:

https://www.arabidopsis.org

The MODOMICS database: http://modomics.genesilico.pl 